June 2011

\title{
A dual formulation of supergravity-matter theories
}

\author{
Daniel Butter and Sergei M. Kuzenko \\ School of Physics M013, The University of Western Australia \\ 35 Stirling Highway, Crawley W.A. 6009, Australia \\ dbutter, kuzenko@cyllene.uwa.edu.au
}

\begin{abstract}
Generating supersymmetric AdS solutions in non-minimal supergravity in four dimensions is notoriously difficult. Indeed, it is a longstanding lore that such solutions exist only for old minimal supergravity. In this paper, we construct a dual formulation for general $\mathcal{N}=1$ supergravity-matter systems that avoids the problem. In the case of pure supergravity without a cosmological constant, it coincides with the usual non-minimal $(n=-1)$ supergravity, but in the presence of matter (or a cosmological constant) our formulation differs considerably. We also elaborate upon the framework of conformal superspace and the compensator method as applied to our theory. In particular, we show that one can encode the details of the Kähler potential and superpotential entirely within the geometry of superspace so that the general sigma-model action is encoded in a single compact term: the supervolume. Finally, we discuss the issue of supercurrents and propose a general form for the supercurrent in AdS.
\end{abstract}




\section{Contents}

1 Introduction 1

2 The improved complex linear multiplet

3 Matter couplings in non-minimal supergravity revisited 4

4 Non-minimal AdS supergravity

5 Conformal supergravity and compensators 11

5.1 A brief survey of $\mathcal{N}=1$ superspace . . . . . . . . . . . . 11

5.2 From conformal superspace to $U(1)$ superspace . . . . . . . . . . . . 15

5.3 From $U(1)$ superspace to conventional superspace . . . . . . . . . . 18

5.4 Old minimal, non-minimal and improved non-minimal supergravity . 21

6 The geometry of matter couplings in non-minimal supergravity 22

7 Concluding remarks and AdS supercurrents 26

A The massless superspin-3/2 multiplet in AdS 31

\section{Introduction}

In a recent paper [1] we have shown that the structure of consistent supercurrents in four-dimensional $\mathcal{N}=1$ anti-de Sitter (AdS) supersymmetry considerably differs from that in the Minkowski case. There are only two irreducible AdS supercurrents, with $12+12$ and $20+20$ degrees of freedom. The former is naturally associated with the so-called longitudinal action $S_{(3 / 2)}^{\|}$for a massless superspin-3/2 multiplet, which involves a real superfield $H_{\alpha \dot{\alpha}}$ and a chiral superfield $\sigma$. The latter is associated with a unique dual formulation $S_{(3 / 2)}^{\perp}$ where the chiral superfield is replaced by a complex linear superfield $\Gamma$. Both these actions represent the lowest superspin limits of two infinite series of dual models for off-shell massless higher spin supermultiplets in AdS [2]. (The actions $S_{(3 / 2)}^{\|}$and $S_{(3 / 2)}^{\perp}$ coincide, respectively, with the actions $S_{\text {old }}$ and $S_{n=-1}$ referred to in the introduction of [1]. Explicit expressions for each are given in Appendix A.) 
The longitudinal formulation $S_{3 / 2}^{\|}$has a natural interpretation in supergravity. It results from the linearization around the AdS background of old minimal $(n=-1 / 3)$ supergravity with a cosmological term. One can show that in the Minkowski limit, $S_{(3 / 2)}^{\perp}$ arises as a linearization of non-minimal $(n=-1)$ supergravity 1 However, there exists a general belief that $S_{(3 / 2)}^{\perp}$ cannot be obtained by linearizing a supergravity action around a supersymmetric AdS solution. This belief can be traced back to 1983 when it was pointed out in [3] that only old minimal supergravity admits a well-defined AdS solution 2 This point was further elaborated upon in [5].

In the present paper, we propose a novel formulation for $n=-1$ non-minimal supergravity and its matter couplings which resolves this puzzle. This formulation provides a simple description of supergravity with a cosmological term such that (i) $\mathcal{N}=1$ AdS superspace is its maximally symmetric solution; and (ii) $S_{(3 / 2)}^{\perp}$ is the linearized action around the AdS background. We also explain how we get around the analysis given in [3].

This paper is organized as follows. In section 2, we describe a globally supersymmetric duality between a chiral multiplet and a complex linear multiplet with a modified constraint. We utilize this simple model in section 3 to describe a new set of matter couplings in non-minimal supergravity. In section 4 , we specialize to the case of pure supergravity with a cosmological constant, demonstrate that AdS is indeed a solution, and describe the way around the "no-go" analysis of [3]. In the remainder of the paper we elaborate upon this construction: in section 5, we discuss in some generality the method of compensators in conformal supergravity and in section 6 , we describe a method to "geometrize" the new matter couplings in the spirit of Kähler superspace [6, 7]. We conclude by briefly discussing supercurrents and consider the case of a non-linear sigma model in AdS. A brief appendix is included discussing the linearized supergravity actions in AdS. Throughout this paper, we shall use the superspace conventions of [4] 3

\footnotetext{
${ }^{1}$ See [3, 4] for reviews of different off-shell versions of $\mathcal{N}=1$ supergravity

${ }^{2}$ Specifically, on page 336 of Superspace [3] Grisaru et al. stated the following. Except for the old minimal formulation of $\mathcal{N}=1$ supergravity, "we find the strange pathologies: de Sitter space cannot be described for $n \neq-1 / 3$ in a globally (de Sitter) supersymmetric way."

${ }^{3}$ These conventions are nearly identical to those of Wess and Bagger [8]. To convert our notation to theirs, one replaces $R \rightarrow 2 R, G_{\alpha \dot{\alpha}} \rightarrow 2 G_{\alpha \dot{\alpha}}$, and $W_{\alpha \beta \gamma} \rightarrow 2 W_{\alpha \beta \gamma}$. In addition, the vector derivative is defined by $\left\{\mathcal{D}_{\alpha}, \overline{\mathcal{D}}_{\dot{\alpha}}\right\}=-2 \mathrm{i} \mathcal{D}_{\alpha \dot{\alpha}}$.
} 


\section{The improved complex linear multiplet}

Let us begin with a simple superconformal model in global supersymmetry involving a chiral superfield $\phi$,

$$
S=\int \mathrm{d}^{4} x \mathrm{~d}^{4} \theta \phi \bar{\phi}, \quad \bar{D}_{\dot{\alpha}} \phi=0
$$

The action is superconformal provided $\phi$ transforms with unit dilatation weight and $U(1)_{R}$ weight $2 / 3.4$ There exists a well understood duality [9, 10] between this model and that involving a complex linear superfield $\Sigma$ obeying the constraint

$$
\bar{D}^{2} \Sigma=0, \quad D^{2} \bar{\Sigma}=0 \text {. }
$$

This constraint is conformally invariant provided $\Sigma$ has dilatation weight $\Delta$ and $U(1)_{R}$ weight $w$ related by

$$
2 \Delta-3 w=4
$$

This condition is solved for a one-parameter family 5 labelled by $n$, with

$$
\Delta=\frac{2}{3 n+1}, \quad w=-\frac{4 n}{3 n+1} .
$$

For $n \neq 0,-1 / 3$, a duality between $\phi$ and $\Sigma$ may be carried out. Our concern here is the case $n=-1$. For that situation, we may introduce the first-order action

$$
S=\int \mathrm{d}^{4} x \mathrm{~d}^{4} \theta\left(\phi \bar{\phi}-\frac{1}{3} \Sigma \phi^{3}-\frac{1}{3} \bar{\Sigma} \bar{\phi}^{3}\right)
$$

where $\phi$ is now an unconstrained complex superfield. The constraint (2.2) is solved by $\Sigma=\bar{D}_{\dot{\alpha}} \bar{\psi}^{\dot{\alpha}}$ for an unconstrained superfield $\bar{\psi}^{\dot{\alpha}}$; therefore, the equation of motion for $\Sigma$ enforces the chirality of $\phi$ and then this action reduces to (2.1). Instead, we may solve the equation of motion for $\phi$ to obtain

$$
\phi^{3}=\Sigma^{-2} \bar{\Sigma}^{-1}
$$

Plugging this in the action yields the dual formulation

$$
S_{\text {dual }}=\frac{1}{3} \int \mathrm{d}^{4} x \mathrm{~d}^{4} \theta(\Sigma \bar{\Sigma})^{-1} .
$$

\footnotetext{
${ }^{4}$ Our convention for the $U(1)_{R}$ weight is such that the derivative $D_{\alpha}$ has weight -1 .

${ }^{5}$ The parameter $n$ was first introduced in the context of non-minimal supergravity [11].
} 
Now let us consider an extension of the action (2.1) by introducing the only possible superconformal interaction term for $\phi$ :

$$
S=\int \mathrm{d}^{4} x \mathrm{~d}^{4} \theta \phi \bar{\phi}-\frac{\mu}{3} \int \mathrm{d}^{4} x \mathrm{~d}^{2} \theta \phi^{3}-\frac{\bar{\mu}}{3} \int \mathrm{d}^{4} x \mathrm{~d}^{2} \bar{\theta} \bar{\phi}^{3}
$$

where $\mu$ is a complex parameter. It is easy to see that this arises from the same first order action

$$
S=\int \mathrm{d}^{4} x \mathrm{~d}^{4} \theta\left(\phi \bar{\phi}-\frac{1}{3} \Gamma \phi^{3}-\frac{1}{3} \bar{\Gamma} \bar{\phi}^{3}\right)
$$

if we require $\Gamma$ to obey the improved complex linear constraint 6

$$
\bar{D}^{2} \Gamma=-4 \mu, \quad D^{2} \bar{\Gamma}=-4 \bar{\mu}
$$

This constraint is indeed superconformally invariant since $\bar{D}^{2} \Gamma$ possesses vanishing dilatation and $U(1)_{R}$ weights. The dual action has then exactly the same form as above, that is

$$
S_{\text {dual }}=\frac{1}{3} \int \mathrm{d}^{4} x \mathrm{~d}^{4} \theta(\Gamma \bar{\Gamma})^{-1}
$$

The constraint (2.10) is responsible for generating the nontrivial interaction terms.

\section{Matter couplings in non-minimal supergravity revisited}

We now consider a locally supersymmetric generalization of the action (2.8) of the form

$$
S=-3 \int \mathrm{d}^{4} x \mathrm{~d}^{4} \theta E \phi \bar{\phi} \mathrm{e}^{-K / 3}+\int \mathrm{d}^{4} x \mathrm{~d}^{2} \theta \mathcal{E} \phi^{3} W+\int \mathrm{d}^{4} x \mathrm{~d}^{2} \bar{\theta} \overline{\mathcal{E}} \bar{\phi}^{3} \bar{W} .
$$

This is the superconformal version (in the spirit of, e.g., Kugo and Uehara [13]) of the most general non-linear sigma model action coupled to supergravity (see, e.g. [8] for a review). The Kähler potential, $K=K\left(\varphi^{i}, \bar{\varphi}^{\bar{j}}\right)$, is a real function of the covariantly chiral superfields $\varphi^{i}$ and their conjugates $\bar{\varphi}^{\bar{j}}$, obeying $\overline{\mathcal{D}}_{\dot{\alpha}} \varphi^{i}=0$. The superpotential, $W=W\left(\varphi^{i}\right)$, is a holomorphic function of $\varphi^{i}$ alone. The conformal compensator $\phi$ is also a covariantly chiral scalar superfield, $\overline{\mathcal{D}}_{\dot{\alpha}} \phi=0$, and it must be nowhere vanishing, $\phi \neq 0$. To describe off-shell supergravity, in this section we use

\footnotetext{
${ }^{6}$ It is of interest to point out that eq. (2.10) also emerges as one of the constraints obeyed by the complex linear Goldstino superfield introduced in [12].
} 
the Wess-Zumino superspace formulation [14] (see [4, 8] for reviews), which at the component level generates the old minimal supergravity multiplet [15].

The action (3.1) is invariant under Kähler transformations,

$$
K \rightarrow K+F+\bar{F}, \quad W \rightarrow \mathrm{e}^{-F} W, \quad \phi \rightarrow \mathrm{e}^{F / 3} \phi
$$

with $F\left(\varphi^{i}\right)$ an arbitrary holomorphic function. Moreover, the action is invariant under super-Weyl transformations [16]

$$
\begin{aligned}
& \mathcal{D}_{\alpha} \rightarrow \mathrm{e}^{\sigma / 2-\bar{\sigma}}\left(\mathcal{D}_{\alpha}-\left(\mathcal{D}^{\beta} \sigma\right) M_{\alpha \beta}\right), \quad \overline{\mathcal{D}}_{\dot{\alpha}} \sigma=0 \\
& \overline{\mathcal{D}}_{\dot{\alpha}} \rightarrow \mathrm{e}^{\bar{\sigma} / 2-\sigma}\left(\overline{\mathcal{D}}_{\dot{\alpha}}-\left(\overline{\mathcal{D}}^{\dot{\beta}} \bar{\sigma}\right) \bar{M}_{\dot{\beta} \dot{\alpha}}\right)
\end{aligned}
$$

which act on the conformal compensator $\phi$ and the matter fields $\varphi^{i}$ as follows:

$$
\begin{aligned}
\phi & \rightarrow \mathrm{e}^{-\sigma} \phi, \\
\varphi^{i} & \rightarrow \varphi^{i} .
\end{aligned}
$$

The standard formulation of this model described, e.g., in [8] may be derived from (3.1) by enforcing the super-Weyl gauge choice $\phi=1$. Then every Kähler transformation must be accompanied by a compensating super-Weyl transformation to maintain the gauge choice $\phi=1$.

We will now perform the very same type of duality transformation as in the previous section. We introduce the first-order action

$$
S=\int \mathrm{d}^{4} x \mathrm{~d}^{4} \theta E\left(-3 \phi \bar{\phi} \mathrm{e}^{-K / 3}+\Gamma \phi^{3}+\bar{\Gamma} \bar{\phi}^{3}\right)
$$

in which $\phi$ is an unconstrained scalar, while the complex scalar $\Gamma$ obeys the improved linear constraint

$$
-\frac{1}{4}\left(\overline{\mathcal{D}}^{2}-4 R\right) \Gamma=W(\varphi)
$$

This constraint is model-dependent 7 For $W=0$ it defines the standard complex linear multiplet.

To maintain Kähler invariance of the action (3.6), $\Gamma$ must transform as

$$
\Gamma \rightarrow \mathrm{e}^{-F} \Gamma
$$

\footnotetext{
${ }^{7}$ In global supersymmetry, constraints of the form (3.7) were introduced for the first time by Deo and Gates [17]. In the context of supergravity, such constraints have recently been used in [12] to generate couplings of the Goldstino superfield to chiral matter.
} 
Since eq. (3.3) implies

$$
\left(\overline{\mathcal{D}}^{2}-4 R\right) \rightarrow \mathrm{e}^{-2 \sigma}\left(\overline{\mathcal{D}}^{2}-4 R\right) \mathrm{e}^{\bar{\sigma}}
$$

when acting on a scalar superfield, and since $W(\varphi)$ is super-Weyl invariant, the superWeyl transformation of $\Gamma$ must be

$$
\Gamma \rightarrow \mathrm{e}^{2 \sigma-\bar{\sigma}} \Gamma
$$

The model introduced above is equivalent to (3.1). Indeed, let us first vary (3.6) with respect to $\Gamma$ using

$$
\delta \Gamma=\overline{\mathcal{D}}_{\dot{\alpha}} \delta \bar{\psi}^{\dot{\alpha}}
$$

with $\delta \bar{\psi}^{\dot{\alpha}}$ an arbitrary spinor superfield. The equation of motion for $\Gamma$ is equivalent to the chirality of $\phi$, and then the first-order action (3.6) turns into (3.1), as a consequence of the standard identity

$$
\int \mathrm{d}^{4} x \mathrm{~d}^{4} \theta E U=-\frac{1}{4} \int \mathrm{d}^{4} x \mathrm{~d}^{2} \theta \mathcal{E}\left(\overline{\mathcal{D}}^{2}-4 R\right) U
$$

and the constraint (3.7). On the other hand, integrating out $\phi$ yields

$$
\phi^{3}=\mathrm{e}^{-K} \Gamma^{-2} \bar{\Gamma}^{-1}
$$

which upon insertion into (3.6) gives the dual action

$$
S_{\text {dual }}=-\int \mathrm{d}^{4} x \mathrm{~d}^{4} \theta E \mathrm{e}^{-K}(\Gamma \bar{\Gamma})^{-1},
$$

which is Kähler invariant. This action provides a novel formulation for general matter couplings in $n=-1$ non-minimal supergravity. The superpotential is present in $S_{\text {dual }}$ via the constraint on $\Gamma$, eq. (3.7).

At this point, it is natural to take a pause and recall the traditional description of matter couplings in the non-minimal supergravity (see textbooks [3, 4] for more detailed presentations). It is well known that any $\mathcal{N}=1$ supergravity-matter system, including the new minimal [18] $(n=0)$ and non-minimal [19, 11] $(n \neq-1 / 3,0)$ supergravity theories, can be realized as a super-Weyl invariant coupling of the old minimal supergravity to matter [20, 4]. To describe the non-minimal supergravity in such a setting, we follow 8 [22, 4] and couple the old minimal supergravity to a complex linear superfield $\Sigma$ and its conjugate $\bar{\Sigma}$,

$$
-\frac{1}{4}\left(\overline{\mathcal{D}}^{2}-4 R\right) \Sigma=0
$$

\footnotetext{
${ }^{8}$ A similar approach was developed by Kugo and Uehara [21].
} 
with the super-Weyl transformation laws

$$
\Sigma \rightarrow \exp \left[\frac{3 n-1}{3 n+1} \sigma-\bar{\sigma}\right] \Sigma
$$

The super-Weyl invariant action for pure non-minimal supergravity is

$$
S_{\text {non-minimal }}=\frac{1}{n} \int \mathrm{d}^{4} x \mathrm{~d}^{4} \theta E(\Sigma \bar{\Sigma})^{(3 n+1) / 2} .
$$

There is a well-elaborated scheme to generalize this action to incorporate matter couplings. To include a superpotential, for instance, we have to deform this action by adding appropriate terms, while keeping the constraint (3.15) fixed. The latter feature is universal. For general supergravity-matter couplings, the constraint (3.15) remains the same.

In our formulation, the superpotential does not appear explicitly in the supergravity matter action (3.14). The superpotential is instead incorporated by appropriately deforming the constraint on $\Gamma$, eq. (3.7). By comparing the super-Weyl transformation laws (3.10) and (3.16), it is seen that our formulation corresponds to $n=-1$.

As shown in [4, 22], the geometric properties of non-minimal supergravity can be described in terms of super-Weyl-inert covariant derivatives, $\mathbb{D}_{A}=\left(\mathbb{D}_{a}, \mathbb{D}_{\alpha}, \overline{\mathbb{D}}^{\dot{\alpha}}\right)$, constructed from the minimal ones, $\mathcal{D}_{A}=\left(\mathcal{D}_{a}, \mathcal{D}_{\alpha}, \overline{\mathcal{D}}^{\dot{\alpha}}\right)$, and a complex conformal compensator $\mathbb{F}$, which transforms under the super-Weyl transformations as

$$
\mathbb{F} \rightarrow \mathbb{F} \mathrm{e}^{-\sigma / 2+\bar{\sigma}}
$$

The explicit construction is as follows:

$$
\begin{gathered}
\mathbb{D}_{\alpha}:=\mathbb{F} \mathcal{D}_{\alpha}-2\left(\mathcal{D}^{\beta} \mathbb{F}\right) M_{\alpha \beta}, \quad \overline{\mathbb{D}}_{\dot{\alpha}}:=\overline{\mathbb{F}} \overline{\mathcal{D}}_{\dot{\alpha}}-2\left(\overline{\mathcal{D}}^{\dot{\beta}} \overline{\mathbb{F}}\right) \bar{M}_{\dot{\beta} \dot{\alpha}}, \\
\mathbb{D}_{\alpha \dot{\alpha}}:=\frac{i}{2}\left\{\mathbb{D}_{\alpha}, \overline{\mathbb{D}}_{\dot{\alpha}}\right\} .
\end{gathered}
$$

They obey the following (anti-)commutation relations:

$$
\begin{aligned}
\left\{\mathbb{D}_{\alpha}, \mathbb{D}_{\beta}\right\}= & -4 \overline{\mathbb{R}} M_{\alpha \beta}, \quad\left\{\overline{\mathbb{D}}_{\dot{\alpha}}, \overline{\mathbb{D}}_{\dot{\beta}}\right\}=4 \mathbb{R} \bar{M}_{\dot{\alpha} \dot{\beta}}, \\
{\left[\overline{\mathbb{D}}_{\dot{\alpha}}, \mathbb{D}_{\beta \dot{\beta}}\right]=} & \frac{1}{2} \varepsilon_{\dot{\alpha} \dot{\beta}} \overline{\mathbb{T}}^{\dot{\gamma}} \mathbb{D}_{\beta \dot{\gamma}}-\mathrm{i} \varepsilon_{\dot{\alpha} \dot{\beta}}\left(\mathbb{R}+\frac{1}{8} \overline{\mathbb{D}}_{\dot{\gamma}} \overline{\mathbb{T}}^{\dot{\gamma}}-\frac{1}{16} \overline{\mathbb{T}}_{\dot{\gamma}} \overline{\mathbb{T}}^{\dot{\gamma}}\right) \mathbb{D}_{\beta} \\
& -\mathrm{i} \varepsilon_{\dot{\alpha} \dot{\beta}}\left(\mathbb{G}_{\beta} \dot{\gamma}-\frac{1}{8} \mathbb{D}_{\beta} \overline{\mathbb{T}}^{\dot{\gamma}}-\frac{1}{8} \overline{\mathbb{D}}^{\dot{\gamma}} \mathbb{T}_{\beta}\right) \overline{\mathbb{D}}_{\dot{\gamma}}-2 \mathrm{i} \varepsilon_{\dot{\alpha} \dot{\beta}} \mathbb{W}_{\beta \gamma \delta} M^{\delta \gamma}+\cdots
\end{aligned}
$$

\footnotetext{
${ }^{9}$ Within the Wess-Zumino superspace formulation, this transformation rule is equivalent to the dilatation and $U(1)_{R}$ weights given in (2.4).
} 
where the ellipsis denotes Lorentz curvature terms. The new torsion superfields are constructed in terms of the old geometric quantities by

$$
\begin{aligned}
\mathbb{T}_{\alpha} & :=\mathbb{D}_{\alpha} \ln \left(\mathbb{F}^{4} \overline{\mathbb{F}}^{2}\right) \\
\mathbb{R} & :=-\frac{1}{4}\left(\overline{\mathcal{D}}^{2}-4 R\right) \overline{\mathbb{F}}^{2}, \\
\mathbb{W}_{\alpha \beta \gamma} & :=\overline{\mathbb{F}}^{2} \mathbb{F} W_{\alpha \beta \gamma} \\
\mathbb{G}_{\alpha \dot{\alpha}} & :=\mathbb{F} \overline{\mathbb{F}} G_{\alpha \dot{\alpha}}-\frac{1}{2}\left(\mathbb{D}_{\alpha} \ln \overline{\mathbb{F}}\right) \overline{\mathbb{D}}_{\dot{\alpha}} \ln \mathbb{F}-\frac{1}{4} \mathbb{D}_{\alpha} \overline{\mathbb{D}}_{\dot{\alpha}} \ln \frac{\overline{\mathbb{F}}^{2}}{\mathbb{F}}+\frac{1}{4} \overline{\mathbb{D}}_{\dot{\alpha}} \mathbb{D}_{\alpha} \ln \frac{\mathbb{F}^{2}}{\overline{\mathbb{F}}} .
\end{aligned}
$$

These new torsion superfields obey a number of Bianchi identities; we do not reproduce them here, but refer the reader to [4]. The structure of torsion superfields uncovered here is essentially that of conformal supergravity, where the dilatations and $U(1)_{R}$ rotations have been "degauged." (The Wess-Zumino derivatives $\mathcal{D}_{A}$ which we have used have an identical algebra but with $\mathbb{T}_{\alpha}=0$.)

To describe non-minimal supergravity, we specialize to a specific compensator $\mathbb{F}$ given in terms of a complex linear superfield $\Sigma$,

$$
\mathbb{F}=\left(\Sigma^{n+1} \bar{\Sigma}^{n-1}\right)^{-(3 n+1) / 8 n} .
$$

Using this relation along with the constraint (3.15), one can check that an additional relation on the torsion is imposed:

$$
\mathbb{R}=-\frac{1}{4}\left(\frac{n+1}{3 n+1}\right) \overline{\mathbb{D}}_{\dot{\alpha}} \overline{\mathbb{T}}^{\dot{\alpha}}+\frac{1}{4}\left(\frac{n+1}{3 n+1}\right)^{2} \overline{\mathbb{T}}_{\dot{\alpha}} \overline{\mathbb{T}}^{\dot{\alpha}}
$$

This equation, we will see in the next section, is why an AdS solution cannot be consistently constructed in non-minimal supergravity.

However, for the case $n=-1$ which concerns us, we shall make use of an improved complex linear compensator, obeying the constraint (3.7). Replacing $\Sigma$ with $\Gamma$ in the above construction, we find that the constraint (3.23) is replaced with

$$
\mathbb{R}=W(\varphi)
$$

This will allow the construction of a non-minimal AdS supergravity, to which we now turn.

\section{Non-minimal AdS supergravity}

The novel formalism for non-minimal $n=-1$ supergravity, which we presented in the previous section, is well-suited to describe supergravity with a cosmological term 
and its AdS solutions. This is achieved by demanding the conformal compensator $\Gamma$ to obey the super-Weyl invariant constraint

$$
-\frac{1}{4}\left(\overline{\mathcal{D}}^{2}-4 R\right) \Gamma=\mu=\mathrm{const}
$$

and choosing the following supergravity action

$$
S_{\mathrm{AdS}}=-\int \mathrm{d}^{4} x \mathrm{~d}^{4} \theta E(\Gamma \bar{\Gamma})^{-1},
$$

which is super-Weyl invariant. Before discussing this theory, it is instructive to recall how $\mathcal{N}=1$ AdS supergravity is described within the old minimal formulation for supergravity (see, e.g., [4] for more details).

AdS supergravity can be described using the super-Weyl invariant action

$$
S_{\text {AdS }}=-3 \int \mathrm{d}^{4} x \mathrm{~d}^{4} \theta E \phi \bar{\phi}+\mu \int \mathrm{d}^{4} x \mathrm{~d}^{2} \theta \mathcal{E} \phi^{3}+\bar{\mu} \int \mathrm{d}^{4} x \mathrm{~d}^{2} \bar{\theta} \overline{\mathcal{E}} \bar{\phi}^{3}
$$

where $\phi$ is the chiral compensator with the super-Weyl transformation law (3.4). This corresponds to (3.1) for the choice $K=0$ and $W=\mu$. The super-Weyl gauge freedom can always be used to fix

$$
\phi=1
$$

and then (4.3) reduces to the standard action for supergravity with cosmological term [3, 4].

Let us analyse the equations of motion corresponding to the theory with action (4.3). The chirality constraint on $\phi$ and its equation of motion can be written, respectively, as

$$
\begin{aligned}
\overline{\mathcal{D}}_{\dot{\alpha}} \phi^{3} & =0 \\
-\frac{1}{4}\left(\overline{\mathcal{D}}^{2}-4 R\right)\left(\bar{\phi} \phi^{-2}\right) & =\mu .
\end{aligned}
$$

We have written these equations in a peculiar way for reasons which will soon be clear. In addition, there is an equation of motion from the gravitational superfield 10 $H^{m}[23,24]$

$$
0=G_{\alpha \dot{\alpha}}+(\phi \bar{\phi})^{1 / 2}\left[\mathcal{D}_{\alpha}, \overline{\mathcal{D}}_{\dot{\alpha}}\right](\phi \bar{\phi})^{-1 / 2}
$$

If we think of (4.3) as a model for chiral matter $\phi$ in a given supergravity background, then the equation (4.6) is simply the condition that the matter supercurrent is equal

\footnotetext{
${ }^{10}$ The gravitational superfield is the sole physical prepotential for conformal supergravity.
} 
to zero. The equations (4.5b) and (4.6) are super-Weyl invariant. In the super-Weyl gauge (4.3), they reduce to

$$
R=\mu, \quad G_{\alpha \dot{\alpha}}=0
$$

which are the equations of motion for $\mathcal{N}=1 \mathrm{AdS}$ supergravity. AdS superspace is a conformally-flat solution of these equations, with a vanishing superconformal Weyl tensor,

$$
W_{\alpha \beta \gamma}=0
$$

Now let us turn to studying the equations of motion corresponding to the nonminimal supergravity action (4.2). The equation of motion for $\Gamma$ and the constraint (4.1) can be written

$$
\begin{aligned}
\overline{\mathcal{D}}_{\dot{\alpha}}\left(\Gamma^{2} \bar{\Gamma}\right)^{-1} & =0 \quad \Longleftrightarrow \quad \overline{\mathbb{T}}_{\dot{\alpha}}=0 \\
-\frac{1}{4}\left(\overline{\mathcal{D}}^{2}-4 R\right) \Gamma & =\mu,
\end{aligned}
$$

with $\overline{\mathbb{T}}_{\dot{\alpha}}$ the conjugate of (3.21a). These equations are equivalent to (4.5) under the duality $\phi^{3} \leftrightarrow 1 / \Gamma^{2} \bar{\Gamma}$. The equation of motion for the gravitational superfield can be shown to be

$$
\mathbb{G}_{\alpha \dot{\alpha}}=0
$$

with $\mathbb{G}_{\alpha \dot{\alpha}}$ the non-minimal curvature (3.21d). This equation is equivalent (using (4.9a)) to

$$
0=G_{\alpha \dot{\alpha}}+(\Gamma \bar{\Gamma})^{-1 / 2}\left[\mathcal{D}_{\alpha}, \overline{\mathcal{D}}_{\dot{\alpha}}\right](\Gamma \bar{\Gamma})^{1 / 2}
$$

It follows from (4.9a) that

$$
\Gamma=\phi^{-2} \bar{\phi}, \quad \overline{\mathcal{D}}_{\dot{\alpha}} \phi=0
$$

on the mass shell. Then, the super-Weyl transformation law (3.10) shows that we can impose the on-shell gauge condition

$$
\Gamma=1 .
$$

In this gauge, the equations (4.9b) and (4.11) turn into the AdS equations (4.7). The AdS solution arises from the choice (4.8). 
It is now easy to explain why the traditional formulation of non-minimal supergravity [3, 4, 5] makes it impossible to generate the AdS geometry

$$
\begin{gathered}
\left\{\mathcal{D}_{\alpha}, \mathcal{D}_{\beta}\right\}=-4 \bar{\mu} M_{\alpha \beta}, \quad\left\{\overline{\mathcal{D}}_{\dot{\alpha}}, \overline{\mathcal{D}}_{\dot{\beta}}\right\}=4 \mu \bar{M}_{\dot{\alpha} \dot{\beta}} \\
\left\{\mathcal{D}_{\alpha}, \overline{\mathcal{D}}_{\dot{\beta}}\right\}=-2 \mathrm{i}\left(\sigma^{c}\right)_{\alpha \dot{\beta}} \mathcal{D}_{c} \equiv-2 \mathrm{i} \mathcal{D}_{\alpha \dot{\beta}} \\
{\left[\mathcal{D}_{a}, \mathcal{D}_{\beta}\right]=-\frac{\mathrm{i}}{2} \bar{\mu}\left(\sigma_{a}\right)_{\beta \dot{\gamma}} \overline{\mathcal{D}^{\dot{\gamma}}}, \quad\left[\mathcal{D}_{a}, \overline{\mathcal{D}}_{\dot{\beta}}\right]=\frac{\mathrm{i}}{2} \mu\left(\sigma_{a}\right)_{\gamma \dot{\beta}} \mathcal{D}^{\gamma}} \\
{\left[\mathcal{D}_{a}, \mathcal{D}_{b}\right]=-|\mu|^{2} M_{a b}}
\end{gathered}
$$

as a globally (AdS) supersymmetric solution. In order to derive (4.14) from nonminimal supergeometry associated with the covariant derivatives defined in (3.19) with the choice (3.22), one has to keep the chiral torsion $\mathbb{R}$ and set to zero the other components of the torsion, including $\mathbb{T}_{\alpha}$ and $\overline{\mathbb{T}}_{\dot{\alpha}}$. But this is contradictory, since for a complex linear compensator $\mathbb{R}$ is constructed in terms of $\overline{\mathbb{T}}_{\dot{\alpha}}$ in accordance with (3.23). If instead we use an improved complex linear compensator (4.1), the torsion $\mathbb{R}$, eq. (3.21b), is identically constant, $\mathbb{R}=\mu$, as a consequence of (3.24).

\section{Conformal supergravity and compensators}

In the preceding sections, we have used the Wess-Zumino superspace formulation involving the covariant derivative $\mathcal{D}_{A}$ and torsion superfields $W_{\alpha \beta \gamma}, R$ and $G_{\alpha \dot{\alpha}}$, which describes the geometry of old minimal $(n=-1 / 3)$ supergravity. This represents the most well-known in a family of different superspace geometries, each well adapted to particular supergravity formulations. Indeed, we have touched briefly upon this in section 3 , where we introduced the covariant derivative $\mathbb{D}_{A}$, with the additional torsion superfield $\mathbb{T}_{\alpha}$, which is well-adapted to describe non-minimal $(n \neq-1 / 3,0)$ supergravities. In this section, we will elaborate upon this construction. We first review the relationship between the various superspace formulations and then discuss how the compensator method can be applied to conformal supergravity to yield them.

\subsection{A brief survey of $\mathcal{N}=1$ superspace}

It was long ago realized at the component level that each of the various supergravity formulations can be understood as conformal supergravity, with $8+8$ degrees of freedom, coupled to various compensators [25] 11 A chiral compensator, with $4+4$ degrees of freedom, yields old minimal $(n=-1 / 3)$ supergravity with $12+12$ components; a real linear compensator, also with $4+4$ components, yields new minimal

\footnotetext{
${ }^{11}$ See also the extensive references in [26] and [27].
} 
$(n=0)$ supergravity; and a complex linear compensator, with $12+12$ components, yields non-minimal $(n \neq-1 / 3,0)$ supergravity with $20+20$ components. Each of these supergravity formulations comes with a corresponding supercurrent multiplet.

The Wess-Zumino superspace geometry, which we used in section 3, describes old minimal supergravity with $12+12$ components. It also economically describes conformal supergravity at the component level. The super-Weyl parameter $\sigma$ contains the component dilatation and $U(1)_{R}$ parameters along with the spinor special conformal parameter (associated with the so-called $S$-supersymmetry). As $\sigma$ possesses $4+4$ components, the effective conformal degrees of freedom in the Wess-Zumino formulation are precisely the $8+8$ components of conformal supergravity. However, $\sigma$ is chiral and so does not represent the most general super-Weyl transformation.

It is possible to enlarge the Wess-Zumino formulation to one which allows an unconstrained complex super-Weyl parameter. This superspace, which was largely developed by Gates and Siegel [11, 28, 29] and which we will refer to as conventional superspace, is very similar to the Wess-Zumino formulation, but involves an additional superfield $T_{\alpha}$. The algebra of covariant derivatives is

$$
\begin{aligned}
\left\{\mathcal{D}_{\beta}, \mathcal{D}_{\alpha}\right\}= & -4 \bar{R} M_{\beta \alpha}, \quad\left\{\overline{\mathcal{D}}^{\dot{\beta}}, \overline{\mathcal{D}}^{\dot{\alpha}}\right\}=4 R \bar{M}^{\dot{\beta} \dot{\alpha}}, \quad\left\{\mathcal{D}_{\alpha}, \overline{\mathcal{D}}_{\dot{\alpha}}\right\}=-2 i \mathcal{D}_{\alpha \dot{\alpha}} \\
{\left[\mathcal{D}_{\beta}, \mathcal{D}_{\alpha \dot{\alpha}}\right]=} & \frac{1}{2} \varepsilon_{\beta \alpha} T^{\gamma} \mathcal{D}_{\gamma \dot{\alpha}}+\mathrm{i} \varepsilon_{\beta \alpha}\left(\bar{R}+\frac{1}{8} \mathcal{D}^{\gamma} T_{\gamma}-\frac{1}{16} T^{\gamma} T_{\gamma}\right) \overline{\mathcal{D}}_{\dot{\alpha}} \\
& -\mathrm{i} \varepsilon_{\beta \alpha}\left(G_{\gamma \dot{\alpha}}+\frac{1}{8} \mathcal{D}_{\gamma} \bar{T}_{\dot{\alpha}}+\frac{1}{8} \overline{\mathcal{D}}_{\dot{\alpha}} T_{\gamma}\right) \mathcal{D}^{\gamma}+\mathrm{i} \overline{\mathcal{D}}_{\dot{\alpha}} \bar{R} M_{\beta \alpha} \\
& -\mathrm{i} \varepsilon_{\beta \alpha}\left(\mathcal{D}_{\delta}-\frac{1}{2} T_{\delta}\right)\left(G_{\gamma \dot{\alpha}}+\frac{1}{8} \mathcal{D}_{\gamma} \bar{T}_{\dot{\alpha}}+\frac{1}{8} \overline{\mathcal{D}}_{\dot{\alpha}} T_{\gamma}\right) M^{\gamma \delta} \\
& +2 \mathrm{i} \varepsilon_{\beta \alpha} \bar{W}_{\dot{\alpha} \dot{\beta} \dot{\gamma}} \bar{M}^{\dot{\beta} \gamma}-2 \mathrm{i} \varepsilon_{\beta \alpha} \bar{Y}^{\dot{\beta}} \bar{M}_{\dot{\beta} \dot{\alpha}}
\end{aligned}
$$

where $\bar{Y}^{\dot{\beta}}$ is a particularly complicated set of derivatives acting upon the superfields $T_{\alpha}$ and $\bar{T}_{\dot{\alpha}} \cdot 12$ This formulation of superspace naturally possesses $24+24$ degrees of freedom at the component level. However, it also may be understood to describe conformal supergravity at the component level. This is because, like the Wess-Zumino formulation, it admits a super-Weyl transformation; but in this case, it is an unconstrained complex super-Weyl transformation [30] acting as

$$
\begin{aligned}
\mathcal{D}_{\alpha}^{\prime} & =L \mathcal{D}_{\alpha}-2 \mathcal{D}^{\beta} L M_{\beta \alpha} \\
T_{\alpha}^{\prime} & =L T_{\alpha}-\mathcal{D}_{\alpha} \ln \left(L^{4} \bar{L}^{2}\right) \\
R^{\prime} & =-\frac{1}{4}\left(\overline{\mathcal{D}}^{2}-4 R\right) \bar{L}^{2} .
\end{aligned}
$$

\footnotetext{
${ }^{12}$ See e.g. 4] for details. $\bar{Y}_{\dot{\alpha}}$ is denoted by $\bar{X}_{\dot{\alpha}}$ in 4$]$.
} 
One may interpret the real part of $\log L$ as a real superfield parameter associated with dilatations and the imaginary part of $\log L$ as a real superfield parameter associated with chiral $U(1)_{R}$ rotations. Since $L$ involves $16+16$ degrees of freedom, there remain $8+8$ conformal degrees of freedom corresponding to the Weyl multiplet of conformal supergravity.

It is possible, due to eq. (5.2b), to choose $L$ such that $T_{\alpha}$ vanishes. Doing so, the algebra above reduces to the Wess-Zumino superspace algebra. One may make residual super-Weyl transformations which preserve the gauge choice $T_{\alpha}=0$ provided $L=\mathrm{e}^{\sigma / 2-\bar{\sigma}}$ for chiral $\sigma$. This is exactly the restricted chiral super-Weyl parameter of the Wess-Zumino superspace formulation. This gauge choice describes old minimal supergravity.

It is also possible, by a suitable super-Weyl transformation, to adopt a gauge where $R$ is defined in terms of $T_{\alpha}$ :

$$
R=-\frac{1}{4}\left(\frac{n+1}{3 n+1}\right) \overline{\mathcal{D}}_{\dot{\alpha}} \bar{T}^{\dot{\alpha}}+\frac{1}{4}\left(\frac{n+1}{3 n+1}\right)^{2} \bar{T}_{\dot{\alpha}} \bar{T}^{\dot{\alpha}} .
$$

Because this choice fixes the components of $R$ in terms of the components of $T_{\alpha}$, the theory has been reduced to $20+20$ degrees of freedom. The resulting superspace structure describes non-minimal supergravity.

However, this formulation does not easily describe new minimal supergravity as it only explicitly gauges the Lorentz group and new minimal supergravity involves the gauging of the $U(1)_{R}$ symmetry. One requires $U(1)$ superspace introduced by Howe [31] (see [3] for a review). Its geometry is described by a covariant derivative $\mathcal{D}_{A}$, now carrying a $U(1)_{R}$ connection, with an algebra

$$
\begin{aligned}
\left\{\mathcal{D}_{\beta}, \mathcal{D}_{\alpha}\right\}= & -4 \bar{R} M_{\beta \alpha}, \quad\left\{\overline{\mathcal{D}}^{\dot{\beta}}, \overline{\mathcal{D}}^{\dot{\alpha}}\right\}=4 R \bar{M}^{\dot{\beta} \dot{\alpha}}, \quad\left\{\mathcal{D}_{\alpha}, \overline{\mathcal{D}}_{\dot{\alpha}}\right\}=-2 \mathrm{i} \mathcal{D}_{\alpha \dot{\alpha}} \\
{\left[\mathcal{D}_{\beta}, \mathcal{D}_{\alpha \dot{\alpha}}\right]=} & +\mathrm{i} \varepsilon_{\beta \alpha} \bar{R} \mathcal{D}_{\dot{\alpha}}-\mathrm{i} \varepsilon_{\beta \alpha} G_{\gamma \dot{\alpha}} \mathcal{D}^{\gamma}+\mathrm{i} \overline{\mathcal{D}}_{\dot{\alpha}} \bar{R} M_{\beta \alpha}-\mathrm{i} \varepsilon_{\beta \alpha} \mathcal{D}_{\delta} G_{\gamma \dot{\alpha}} M^{\gamma \delta} \\
& +2 \mathrm{i} \varepsilon_{\beta \alpha} \bar{W}_{\dot{\alpha} \dot{\beta} \dot{\gamma}} \bar{M}^{\dot{\beta} \gamma}-\frac{\mathrm{i}}{3} \varepsilon_{\beta \alpha} \bar{X}^{\dot{\beta}} \bar{M}_{\dot{\beta} \dot{\alpha}}+\frac{\mathrm{i}}{2} \varepsilon_{\beta \alpha} \bar{X}_{\dot{\alpha}} \mathbf{A} .
\end{aligned}
$$

The superfields $R, G_{\alpha \dot{\alpha}}, W_{\alpha \beta \gamma}$, and $X_{\alpha}$ obey the relations

$$
\begin{aligned}
& \overline{\mathcal{D}}_{\dot{\alpha}} R=0, \quad \overline{\mathcal{D}}_{\dot{\alpha}} X_{\alpha}=0, \quad \overline{\mathcal{D}}_{\dot{\alpha}} W_{\alpha \beta \gamma}=0 \\
& X_{\alpha}=\mathcal{D}_{\alpha} R-\overline{\mathcal{D}}^{\dot{\alpha}} G_{\alpha \dot{\alpha}},
\end{aligned}
$$

The $U(1)_{R}$ generator $\mathbf{A}$ is normalized such that

$$
\left[\mathbf{A}, \mathcal{D}_{\alpha}\right]=-\mathcal{D}_{\alpha}, \quad\left[\mathbf{A}, \overline{\mathcal{D}}_{\dot{\alpha}}\right]=+\overline{\mathcal{D}}_{\dot{\alpha}}
$$


The corresponding $U(1)_{R}$ transformations are gauged with all objects, including the torsion superfields transforming with definite $U(1)_{R}$ charge. In addition, one has an ungauged super-Weyl transformation given by

$$
\mathcal{D}_{\alpha}^{\prime}=\mathrm{e}^{\Lambda / 2}\left(\mathcal{D}_{\alpha}+2 \mathcal{D}^{\beta} \Lambda M_{\beta \alpha}+\frac{3}{2} \mathcal{D}_{\alpha} \Lambda \mathbf{A}\right)
$$

The algebra (5.4) and relations (5.5) are much simpler than in the conventional formulation. The price one pays for this simplification is that this superspace can only describe $U(1)_{R}$-invariant theories.

The geometry naturally possesses $16+16$ degrees of freedom. To describe new minimal supergravity, one can perform a super-Weyl transformation to the gauge $R=0$. This reduces the theory to $12+12$ components and corresponds to new minimal supergravity.

It is possible to derive conventional superspace from $U(1)$ superspace. One performs a "degauging" procedure where the $U(1)_{R}$ connection is removed from the covariant derivative and reinterpreted as a torsion superfield. This is, in a certain sense, the origin of $T_{\alpha}$ : it is "really" the spinor $U(1)_{R}$ connection $A_{\alpha}$ of $U(1)$ superspace. In order to maintain the simple forms of the spinor anti-commutators in (5.4), one must redefine the superfield $R$ and the Lorentz connection (see, e.g., the discussion in [3]). These lead to the more complicated algebra (5.1). Moreover, after making these redefinitions, one may show that the (now) ungauged $U(1)_{R}$ transformation combines with the real super-Weyl transformation to yield the complex super-Weyl transformation (5.2).

In proceeding from the conventional superspace to $U(1)$ superspace, we have gauged half of the original complex super-Weyl parameter. It is natural to ask whether it is possible to gauge the remainder? It turns out the answer is yes. However, instead of just enlarging the structure group with dilatations, one must also gauge special superconformal transformations, involving not just the special conformal generator $K_{a}$ but also the $S$-supersymmetry generators $S_{\alpha}$ and $\bar{S}^{\dot{\alpha}}$. The resulting superspace is called conformal superspace [32]. Its geometry is characterized by a covariant derivative $\nabla_{A}$ constructed out of connections valued in the full superconformal algebra. It turns out that the algebra of the covariant derivatives is quite simple, with all curvatures constructed solely from the superconformal Weyl curvature $W_{\alpha \beta \gamma}$. One finds 13

\footnotetext{
${ }^{13}$ We remind the reader that we make use of the conventions of [4] for the Lorentz generator and normalization of $W_{\alpha \beta \gamma}$. These conventions are different from those originally employed in 32 .
} 


$$
\begin{gathered}
\left\{\nabla_{\alpha}, \nabla_{\beta}\right\}=\left\{\bar{\nabla}_{\dot{\alpha}}, \bar{\nabla}_{\dot{\beta}}\right\}=0, \quad \nabla_{\alpha \dot{\alpha}}:=\frac{\mathrm{i}}{2}\left\{\nabla_{\alpha}, \bar{\nabla}_{\dot{\alpha}}\right\} \\
{\left[\nabla_{\beta}, \nabla_{\alpha \dot{\alpha}}\right]=2 i \varepsilon_{\beta \alpha} \bar{W}_{\dot{\alpha} \dot{\beta} \dot{\gamma}} \bar{M}^{\dot{\beta} \dot{\gamma}}-R(\bar{S})_{\beta \alpha \dot{\alpha} \dot{\gamma}} \bar{S}^{\dot{\gamma}}-R(K)_{\beta \alpha \dot{\alpha}}{ }^{c} K_{c}}
\end{gathered}
$$

where $R(\bar{S})_{\beta \alpha \dot{\alpha} \dot{\gamma}}$ and $R(K)_{\beta \alpha \dot{\alpha}}{ }^{c}$ involve derivatives of the superfield $\bar{W}_{\dot{\alpha} \dot{\beta} \dot{\gamma}}$; their precise forms will not be necessary for our discussion. The algebra of these derivatives is considerably simplified while at the same time the structure group has enlarged. All symmetries are gauged with only the $8+8$ degrees of freedom of conformal supergravity surviving at the component level. Of course, this huge simplification comes at a high cost: only superconformally invariant actions can be described in conformal superspace.

Just as conventional superspace may be derived by degauging $U(1)$ superspace, the latter may be derived by degauging conformal superspace. Relative to $U(1)$ superspace, there are additional connections associated with dilatations and the special superconformal transformations. The special superconformal symmetry may be used to eliminate the dilatation connection algebraically. The residual special superconformal connections can be shown to yield precisely $R, G_{\alpha \dot{\alpha}}$ and $X_{\alpha}$ of $U(1)$ superspace. The details are given in [32].

The degauging procedure which takes one from conformal superspace to $U(1)$ superspace to conventional superspace is straightforward but messy. It requires specific gauges to be imposed along the way and specific redefinitions of connections to be made to keep the spinor anti-commutators simple. A far more elegant method is available, which is similar to that used in section 3 where we reviewed how to use an explicit compensator to convert the Wess-Zumino formulation of superspace to conventional superspace (and its non-minimal formulation in particular). We would like now to apply the same idea to the construction of conventional superspace directly from conformal superspace. It is natural to consider this procedure in two steps: deriving $U(1)$ superspace first and then conventional superspace from $U(1)$.

\subsection{From conformal superspace to $U(1)$ superspace}

Let us assume that conformal superspace is furnished with a real, nowhere-vanishing superfield $X=\exp U$ with non-vanishing dilatation weight, which we assume to be 2 
without loss of generality. We define new derivatives $\hat{\mathcal{D}}_{A}$ by 14

$$
\begin{aligned}
\hat{\mathcal{D}}_{\alpha} & :=\nabla_{\alpha}-\frac{1}{2} \nabla_{\alpha} U \mathbf{D}-\nabla^{\beta} U M_{\beta \alpha}-\frac{3}{4} \nabla_{\alpha} U \mathbf{A} \\
\overline{\hat{\mathcal{D}}}_{\dot{\alpha}} & :=\bar{\nabla}_{\dot{\alpha}}-\frac{1}{2} \bar{\nabla}_{\dot{\alpha}} U \mathbf{D}-\bar{\nabla}^{\dot{\beta}} U \bar{M}_{\dot{\beta} \dot{\alpha}}+\frac{3}{4} \bar{\nabla}_{\dot{\alpha}} U \mathbf{A} \\
\hat{\mathcal{D}}_{\alpha \dot{\alpha}} & :=\frac{\mathrm{i}}{2}\left\{\hat{\mathcal{D}}_{\alpha}, \overline{\hat{\mathcal{D}}}_{\dot{\alpha}}\right\},
\end{aligned}
$$

where $\mathbf{D}$ and $\mathbf{A}$ are the dilatation and $U(1)_{R}$ generators, normalized such that

$$
\begin{aligned}
{\left[\mathbf{D}, \nabla_{\alpha}\right]=\frac{1}{2} \nabla_{\alpha}, } & {\left[\mathbf{D}, \bar{\nabla}_{\dot{\alpha}}\right] } & =\frac{1}{2} \bar{\nabla}_{\dot{\alpha}} \\
{\left[\mathbf{A}, \nabla_{\alpha}\right]=-\nabla_{\alpha}, } & {\left[\mathbf{A}, \bar{\nabla}_{\dot{\alpha}}\right]=} & +\bar{\nabla}_{\dot{\alpha}} .
\end{aligned}
$$

The new derivatives $\hat{\mathcal{D}}_{A}$ have the same dilatation and $U(1)_{R}$ weights as $\nabla_{A}$. However, unlike $\nabla_{A}$, they have the property that they act on conformally primary superfields to give new conformally primary superfields. Recall that a superfield $\Psi$ is called primary if it is annihilated by the special superconformal generators,

$$
K_{B} \Psi=0, \quad K_{B}=\left(K_{b}, S_{\beta}, \bar{S}^{\dot{\beta}}\right)
$$

One can show that the derivative of $\Psi$, constructed using $\hat{\mathcal{D}}_{A}$, is also primary,

$$
K_{B} \Psi=0 \Longrightarrow K_{B} \hat{\mathcal{D}}_{A} \Psi=0
$$

This requirement uniquely determines the definition of $\hat{\mathcal{D}}_{\alpha}$ and $\overline{\hat{\mathcal{D}}}_{\dot{\alpha}}$ in (15.9). As we will show, they are closely related to the super-Weyl invariant derivatives constructed in [22] 15 Note that if we are careful to only work with conformally primary superfields $\Psi$ and their derivatives $\hat{\mathcal{D}}_{A} \Psi$, then the special superconformal generators appearing in $\hat{\mathcal{D}}_{A}$ are purely spectator connections. In particular, when we discuss the curvatures $\left[\hat{\mathcal{D}}_{A}, \hat{\mathcal{D}}_{B}\right]$, we will always assume that we are acting on conformally primary superfields and so we can always ignore the special superconformal curvatures.

There is another key feature of these new derivatives. The compensator $X$ is covariantly constant with respect to them,

$$
\hat{\mathcal{D}}_{\alpha} X=\overline{\hat{\mathcal{D}}}_{\dot{\alpha}} X=0
$$

This implies that

$$
\left[\hat{\mathcal{D}}_{A}, \hat{\mathcal{D}}_{B}\right] X=0
$$

\footnotetext{
${ }^{14}$ These new derivatives were constructed originally by Kugo and Uehara at the level of tensor calculus in their treatment of conformal supergravity [21]. Their present form appeared in [33].

${ }^{15}$ See also the discussion in [4] for a pedagogical review.
} 
and so the only curvatures present in $\left[\hat{\mathcal{D}}_{A}, \hat{\mathcal{D}}_{B}\right]$ must be those for which $X$ is a scalar. Thus we are left with torsion, Lorentz, and $U(1)_{R}$ curvatures; there can be no dilatation curvatures. In fact, one can show that when these new derivatives act only on conformally primary objects, they obey the same algebra as (5.4)

$$
\begin{aligned}
& \left\{\hat{\mathcal{D}}_{\beta}, \hat{\mathcal{D}}_{\alpha}\right\}=-4 \overline{\hat{R}} M_{\beta \alpha}, \quad\left\{\overline{\hat{\mathcal{D}}}^{\dot{\beta}}, \overline{\hat{\mathcal{D}}}^{\dot{\alpha}}\right\}=4 \hat{R} \bar{M}^{\dot{\beta} \dot{\alpha}} \\
& {\left[\hat{\mathcal{D}}_{\beta}, \hat{\mathcal{D}}_{\alpha \dot{\alpha}}\right]=-\mathrm{i} \varepsilon_{\beta \alpha} \hat{G}_{\gamma \dot{\alpha}} \hat{\mathcal{D}}^{\gamma}+\mathrm{i} \varepsilon_{\beta \alpha} \overline{\hat{R}} \hat{\mathcal{D}}_{\dot{\alpha}}-\mathrm{i} \varepsilon_{\beta \alpha} \hat{\mathcal{D}}_{\delta} \hat{G}_{\gamma \dot{\alpha}} M^{\gamma \delta}+\mathrm{i} \overline{\mathcal{D}}_{\dot{\alpha}} \overline{\hat{R}} M_{\beta \alpha}} \\
& +2 \mathrm{i} \varepsilon_{\beta \alpha} \bar{W}_{\dot{\alpha} \dot{\beta} \dot{\gamma}} \bar{M}^{\dot{\beta} \gamma}-\frac{\mathrm{i}}{3} \varepsilon_{\beta \alpha} \overline{\hat{X}}^{\dot{\beta}} \bar{M}_{\dot{\beta} \dot{\alpha}}+\frac{\mathrm{i}}{2} \varepsilon_{\beta \alpha} \overline{\hat{X}}_{\dot{\alpha}} \mathbf{A} \text {. }
\end{aligned}
$$

where we have defined

$$
\hat{R}:=-\frac{1}{4 X} \bar{\nabla}^{2} X, \quad \hat{G}_{\alpha \dot{\alpha}}:=X^{1 / 2}\left[\nabla_{\alpha}, \bar{\nabla}_{\dot{\alpha}}\right] X^{-1 / 2}, \quad \hat{X}_{\alpha}:=\frac{3}{4} \bar{\nabla}^{2} \nabla_{\alpha} \log X .
$$

The superfield $W_{\alpha \beta \gamma}$ is identical to that appearing in the original conformal superspace algebra (5.8). Each of the new torsion superfields is conformally primary. They can be shown to obey the constraints

$$
\begin{aligned}
& \overline{\hat{\mathcal{D}}}_{\dot{\alpha}} \hat{R}=0, \quad \overline{\hat{\mathcal{D}}}_{\dot{\alpha}} \hat{X}_{\alpha}=0, \quad \overline{\hat{\mathcal{D}}}_{\dot{\alpha}} W_{\alpha \beta \gamma}=0 \\
& \hat{X}_{\alpha}=\hat{\mathcal{D}}_{\alpha} \hat{R}-\overline{\hat{\mathcal{D}}}^{\dot{\alpha}} \hat{G}_{\alpha \dot{\alpha}}, \quad \hat{\mathcal{D}}^{\alpha} \hat{X}_{\alpha}=\overline{\mathcal{D}}_{\dot{\alpha}} \overline{\hat{X}}^{\dot{\alpha}}
\end{aligned}
$$

which are identical in form to (5.5). This structure is exactly that of $U(1)$ superspace.

One may make a further set of redefinitions which clarifies that dilatations have essentially been removed from the structure group along with special conformal transformations. For every superfield $\Psi$ (including our torsion superfields) with dilatation weight $\Delta$, we may define a new superfield $\boldsymbol{\Psi}$ by

$$
\Psi:=\mathrm{e}^{-U \mathbf{D} / 2} \mathcal{F}=X^{-\Delta / 2} \Psi
$$

which is inert under superfield dilatations. For our torsion superfields, this amounts to

$$
\begin{array}{r}
\mathbf{R}:=X^{-1 / 2} \hat{R}=-\frac{1}{4 X^{3 / 2}} \bar{\nabla}^{2} X, \quad \mathbf{G}_{\alpha \dot{\alpha}}:=X^{-1 / 2} \hat{G}_{\alpha \dot{\alpha}}=\left[\nabla_{\alpha}, \bar{\nabla}_{\dot{\alpha}}\right] X^{-1 / 2} \\
\mathbf{X}_{\alpha}:=X^{-3 / 4} \hat{X}_{\alpha}=\frac{3}{4} X^{-3 / 4} \bar{\nabla}^{2} \nabla_{\alpha} \log X, \quad \mathbf{W}_{\alpha \beta \gamma}:=X^{-3 / 4} W_{\alpha \beta \gamma} .
\end{array}
$$

Similarly, the operator $\hat{\mathcal{D}}_{\alpha}$, which carries dilatation weight $1 / 2$, may be used to define a new operator $\mathcal{D}_{\alpha}$ via the similarity transformation

$$
\mathcal{D}_{\alpha}:=\mathrm{e}^{-U \mathbf{D} / 2} \hat{\mathcal{D}}_{\alpha} \mathrm{e}^{U \mathbf{D} / 2}=X^{-1 / 4}\left(\nabla_{\alpha}-\nabla^{\beta} U M_{\beta \alpha}-\frac{3}{4} \nabla_{\alpha} U \mathbf{A}\right)
$$


These new derivatives also obey the $U(1)$ superspace algebra, but with all the objects modified by the similarity transformation.

These new objects are inert under the dilatations of conformal superspace; we say that the superfield $X$ has compensated for the dilatations. However, there is a new transformation that the compensated objects possess. If we consider a finite shift in the compensator $X$

$$
X \rightarrow \mathrm{e}^{-2 \Lambda} X
$$

the new covariant derivative transforms as

$$
\mathcal{D}_{\alpha} \rightarrow \mathrm{e}^{\Lambda / 2}\left(\mathcal{D}_{\alpha}+2 \mathcal{D}^{\beta} \Lambda M_{\beta \alpha}+\frac{3}{2} \mathcal{D}_{\alpha} \Lambda \mathbf{A}\right)
$$

This is exactly the super-Weyl transformation of $U(1)$ superspace. It manifests itself here from an explicit shift in the compensator.

This additional similarity transformation is quite natural since it absorbs the compensator into the frame of superspace by the redefinition of the vierbein implicit in (5.20). For example, given a superconformal action

$$
S=\int \mathrm{d}^{4} x \mathrm{~d}^{4} \theta E X^{1-\Delta / 2} \mathcal{L}
$$

where $\mathcal{L}$ is some quantity with dilatation weight $\Delta$, the derivatives $\hat{\mathcal{D}}_{A}$ treat $X$ as if it were constant. Under the similarity transformation, the action becomes

$$
S=\int \mathrm{d}^{4} x \mathrm{~d}^{4} \theta \boldsymbol{E} \mathcal{L}
$$

with all explicit factors of $X$ being absorbed either into other superfields or the frame of superspace. This agrees with the general notion of a compensator field as discussed in [3] where a compensator field is explicitly used to construct the vierbein.

The geometry of $U(1)$ superspace is well-adapted to describe new minimal supergravity constructed with a linear compensator. However, since our concern is with old minimal and non-minimal geometry, we will proceed to degauge to conventional superspace.

\subsection{From $U(1)$ superspace to conventional superspace}

Now we turn to the task of degauging the $U(1)_{R}$ connection. The simplest way of achieving this is again via the use of an explicit compensator field. In analogy 
to the real compensator $X$ which transforms under dilatations with some weight, we could introduce a real compensator, say $Y$, transforming under $U(1)_{R}$ chiral rotations with some weight. However, describing the geometry in terms of two separate real compensators is somewhat artificial. It is more natural to consider a single complex superfield $\mathbb{F}$ with non-vanishing dilatation and $U(1)_{R}$ weights. Without loss of generality, we take those to be $-1 / 2$ and 1 , so that the dilatation compensator $X$ used in $U(1)$ superspace is given by

$$
X=(\mathbb{F} \overline{\mathbb{F}})^{-2}
$$

The reason for this choice is so that subsequent formulae simplify.

It turns out that the presence of both dilatation and $U(1)_{R}$ compensators offers a new dimension- $1 / 2$ conformally primary superfield in addition to the superfields defined previously. This combination, which we denote $\hat{\mathbb{T}}_{\alpha}$, can be written in terms of the $U(1)$ superspace spinor derivative $\hat{\mathcal{D}}_{\alpha}$ as

$$
\hat{\mathbb{T}}_{\alpha}:=\hat{\mathcal{D}}_{\alpha} \log (\mathbb{F} / \overline{\mathbb{F}})
$$

Equivalently, we can write this quantity using the original conformal superspace derivative as

$$
\hat{\mathbb{T}}_{\alpha}=\nabla_{\alpha} \log \left(\mathbb{F}^{4} \overline{\mathbb{F}}^{2}\right)
$$

It is now possible to degauge the $U(1)_{R}$ symmetry by introducing the new covariant derivatives

$$
\begin{aligned}
\hat{\mathbb{D}}_{\alpha} & :=\hat{\mathcal{D}}_{\alpha}-\hat{\mathbb{T}}^{\beta} M_{\beta \alpha}-\frac{1}{2} \hat{\mathbb{T}}_{\alpha} \mathbf{A} \\
\hat{\overline{\mathbb{D}}}_{\dot{\alpha}} & :=\hat{\mathcal{D}}_{\dot{\alpha}}-\overline{\hat{\mathbb{T}}}^{\dot{\beta}} \bar{M}_{\dot{\beta} \dot{\alpha}}+\frac{1}{2} \overline{\hat{\mathbb{T}}}_{\dot{\alpha}} \mathbf{A} \\
\hat{\mathbb{D}}_{\alpha \dot{\alpha}} & :=\frac{i}{2}\left\{\hat{\mathbb{D}}_{\alpha}, \overline{\hat{\mathbb{D}}}_{\dot{\alpha}}\right\} .
\end{aligned}
$$

The modification of the $U(1)_{R}$ connection is necessary so that the new derivatives obey

$$
\hat{\mathbb{D}}_{A} \mathbb{F}=0
$$

which guarantees that no $U(1)_{R}$ curvatures will appear in the (anti-)commutator $\left[\hat{\mathbb{D}}_{A}, \hat{\mathbb{D}}_{B}\right]$. Unlike in the previous section, there is a certain degree of arbitrariness in the definitions made for $\hat{\mathbb{D}}$ - specifically, in the choice of how to deform the Lorentz connection. The reason for this is that $\hat{\mathbb{T}}_{\alpha}$ is already conformally primary, so there 
is no unambiguous choice for $\hat{\mathbb{D}}_{A}$. The coefficient we have chosen is so that the anti-commutator of $\hat{\mathbb{D}}_{\alpha}$ with itself is simply

$$
\left\{\hat{\mathbb{D}}_{\alpha}, \hat{\mathbb{D}}_{\beta}\right\}=-4 \overline{\hat{\mathbb{R}}} M_{\beta \alpha}, \quad\left\{\hat{\overline{\mathbb{D}}}_{\dot{\alpha}}, \hat{\overline{\mathbb{D}}}_{\dot{\beta}}\right\}=+4 \hat{\mathbb{R}} \bar{M}_{\dot{\beta} \dot{\alpha}}
$$

where

$$
\hat{\mathbb{R}}:=\hat{R}-\frac{1}{4} \overline{\hat{\mathcal{D}}}_{\dot{\alpha}} \overline{\hat{\mathrm{T}}}^{\dot{\alpha}}-\frac{1}{4} \overline{\hat{\mathbb{T}}}_{\dot{\alpha}} \overline{\hat{\mathbb{T}}}^{\dot{\alpha}}
$$

Any other choice would introduce dimension-1/2 torsion. In [3] this is understood as a certain arbitrariness in how one chooses to define the spin connection, or equivalently, an arbitrariness in what specific constraints to place upon the torsion. For the remainder of the (anti-)commutators, we find a result identical to that given in (3.20) and (5.1) provided we make the following definition for the superfield $\hat{\mathbb{G}}_{\alpha \dot{\alpha}}$ :

$$
\hat{\mathbb{G}}_{\alpha \dot{\alpha}}:=\hat{G}_{\alpha \dot{\alpha}}-\frac{3}{8} \hat{\mathcal{D}}_{\alpha} \overline{\hat{\mathbb{T}}}_{\dot{\alpha}}+\frac{3}{8} \overline{\hat{\mathcal{D}}}_{\dot{\alpha}} \hat{\mathbb{T}}_{\alpha}+\frac{1}{4} \hat{\mathbb{T}}_{\alpha} \overline{\hat{\mathbb{T}}}_{\dot{\alpha}} \text {. }
$$

This quantity may be expressed in terms of conformal superspace derivatives and the explicit compensators as

$$
\begin{aligned}
\hat{\mathbb{G}}_{\alpha \dot{\alpha}}= & -\frac{1}{4} \nabla_{\alpha} \bar{\nabla}_{\dot{\alpha}} \log \frac{\overline{\mathbb{F}}^{2}}{\mathbb{F}}+\frac{1}{4} \bar{\nabla}_{\dot{\alpha}} \nabla_{\alpha} \log \frac{\mathbb{F}^{2}}{\overline{\mathbb{F}}} \\
& -\frac{1}{4} \nabla_{\alpha} \log (\mathbb{F} \overline{\mathbb{F}}) \bar{\nabla}_{\dot{\alpha}} \log (\mathbb{F} \overline{\mathbb{F}})-\frac{1}{4} \nabla_{\alpha} \log (\mathbb{F} / \overline{\mathbb{F}}) \bar{\nabla}_{\dot{\alpha}} \log (\mathbb{F} / \overline{\mathbb{F}})
\end{aligned}
$$

In a similar vein, the superfield $\hat{\mathbb{R}}$ may be written as

$$
\hat{\mathbb{R}}=-\frac{1}{4} \overline{\mathbb{F}}^{-2} \bar{\nabla}^{2} \overline{\mathbb{F}}^{2}
$$

As in our construction of $U(1)_{R}$ superspace, these new derivatives and superfields are not exactly those of conventional superspace. One must perform an additional similarity transformation involving both the dilatation and $U(1)_{R}$ compensators on all operators and superfields to remove the dilatation and $U(1)_{R}$ connections completely. For the covariant derivative, we find

$$
\mathbb{D}_{\alpha}=\mathbb{F} \nabla_{\alpha}-2 \nabla^{\beta} \mathbb{F} M_{\beta \alpha}
$$

after the appropriate similarity transformation. The torsion superfields are given by

$$
\begin{aligned}
\mathbb{T}_{\alpha}= & \mathbb{F} \nabla_{\alpha} \log \left(\mathbb{F}^{4} \overline{\mathbb{F}}^{2}\right) \\
\mathbb{R}= & -\frac{1}{4} \bar{\nabla}^{2} \overline{\mathbb{F}}^{2} \\
\mathbb{G}_{\alpha \dot{\alpha}}= & -\frac{1}{4} \mathbb{F} \overline{\mathbb{F}} \nabla_{\alpha} \bar{\nabla}_{\dot{\alpha}} \log \frac{\overline{\mathbb{F}}^{2}}{\mathbb{F}}+\frac{1}{4} \overline{\mathbb{F}} \overline{\mathbb{F}} \bar{\nabla}_{\dot{\alpha}} \nabla_{\alpha} \log \frac{\mathbb{F}^{2}}{\overline{\mathbb{F}}} \\
& \quad-\frac{1}{4} \mathbb{F} \overline{\mathbb{F}} \nabla_{\alpha} \log (\mathbb{F} \overline{\mathbb{F}}) \bar{\nabla}_{\dot{\alpha}} \log (\mathbb{F} \overline{\mathbb{F}})-\frac{1}{4} \mathbb{F} \overline{\mathbb{F}} \nabla_{\alpha} \log (\mathbb{F} / \overline{\mathbb{F}}) \bar{\nabla}_{\dot{\alpha}} \log (\mathbb{F} / \overline{\mathbb{F}}) \\
\mathbb{W}_{\alpha \beta \gamma}= & \overline{\mathbb{F}}^{2} \mathbb{F} W_{\alpha \beta \gamma} .
\end{aligned}
$$


If we consider arbitrary redefinitions of the compensator,

$$
\mathbb{F} \rightarrow L \mathbb{F}
$$

we find the complex super-Weyl transformations

$$
\begin{aligned}
\mathbb{D}_{\alpha} & \rightarrow L \mathbb{D}_{\alpha}-2 \mathbb{D}^{\beta} L M_{\beta \alpha} \\
\mathbb{T}_{\alpha} & \rightarrow L \mathbb{T}_{\alpha}+\mathbb{D}_{\alpha} \log \left(L^{4} \bar{L}^{2}\right) \\
\mathbb{R} & \rightarrow-\frac{1}{4}\left(\overline{\mathbb{D}}^{2}-4 \mathbb{R}\right) \bar{L}^{2} .
\end{aligned}
$$

In this formulation, all operators and superfields have been rendered completely inert under both dilatations and the $U(1)_{R}$ rotations. In their place we are left with a complex super-Weyl transformation arising from shifts in the complex compensator $\mathbb{F}$.

\subsection{Old minimal, non-minimal and improved non-minimal supergravity}

There are two immediate choices of complex compensator which can be made. The most obvious choice is a chiral compensator. If we choose $\mathbb{F}=\phi^{1 / 2} \bar{\phi}^{-1}$, we immediately observe that

$$
\mathbb{T}_{\alpha}=\overline{\mathbb{T}}_{\dot{\alpha}}=0
$$

The geometry of Wess and Zumino which we have used in sections 2 through 4 corresponds to such a conventional superspace description.

A more interesting possibility is offered by the family of non-minimal supergravities. These coincide with the choice

$$
\mathbb{F}=\left(\Sigma^{n+1} \bar{\Sigma}^{n-1}\right)^{-(3 n+1) / 8 n}
$$

for a complex linear multiplet. Recall that the dilatation and $U(1)_{R}$ weights of $\Sigma$ are parametrized by a single real number $n$,

$$
\Delta=\frac{2}{3 n+1}, \quad w=-\frac{4 n}{3 n+1}, \quad n \neq-1 / 3,0 .
$$

Now, because of the constraint $\bar{\nabla}^{2} \Sigma=0$ one can show that the superfield $\mathbb{R}$ is actually given in terms of $\overline{\mathbb{T}}_{\dot{\alpha}}$ as

$$
\mathbb{R}=-\frac{1}{4}\left(\frac{n+1}{3 n+1}\right) \overline{\mathbb{D}}_{\dot{\alpha}} \overline{\mathbb{T}}^{\dot{\alpha}}+\frac{1}{4}\left(\frac{n+1}{3 n+1}\right)^{2} \overline{\mathbb{T}}_{\dot{\alpha}} \overline{\mathbb{T}}^{\dot{\alpha}} .
$$


This relation defines the so-called non-minimal superspaces.

Finally, we consider the case where the superfield $\Gamma$ obeys the improved complex linearity constraint

$$
-\frac{1}{4} \bar{\nabla}^{2} \Gamma=Q
$$

where $Q$ is some chiral superfield. We will be most interested in the case where it is a holomorphic function of some chiral matter superfields, but for the moment we will allow it to be arbitrary. $\Gamma$ has dilatation and $U(1)_{R}$ weights parametrized by a real number $n$ (5.41) and so $Q$ must have dilatation and $U(1)_{R}$ weights given by

$$
\Delta(Q)=3\left(\frac{n+1}{3 n+1}\right), \quad w(Q)=2\left(\frac{n+1}{3 n+1}\right) .
$$

Note that $\Delta(Q)$ and $w(Q)$ are in the ratio of $3: 2$ as required for $Q$ to be chiral. One can show that in this situation, the constraint (5.42) is modified to

$$
\mathbb{R}=\mathbb{Q}-\frac{1}{4}\left(\frac{n+1}{3 n+1}\right) \overline{\mathbb{D}}_{\dot{\alpha}} \overline{\mathbb{T}}^{\dot{\alpha}}+\frac{1}{4}\left(\frac{n+1}{3 n+1}\right)^{2} \overline{\mathbb{T}}_{\dot{\alpha}} \overline{\mathbb{T}}^{\dot{\alpha}},
$$

where $\mathbb{Q}$ is the superfield $Q$ dressed with the appropriate factors of the compensator $\mathbb{F}$ to render it inert,

$$
\mathbb{Q}:=(\mathbb{F} \overline{\mathbb{F}})^{\Delta(Q)}\left(\frac{\overline{\mathbb{F}}}{\overline{\mathbb{F}}}\right)^{w(Q) / 2} Q
$$

\section{The geometry of matter couplings in non-minimal supergravity}

We turn now to our final topic: the geometrization of the dual action (3.14), which for convenience we repeat here

$$
S_{\text {dual }}=-\int \mathrm{d}^{4} x \mathrm{~d}^{4} \theta E \mathrm{e}^{-K}(\Gamma \bar{\Gamma})^{-1}
$$

The superfield $\Gamma$ obeys the constraint

$$
-\frac{1}{4}\left(\overline{\mathcal{D}}^{2}-4 R\right) \Gamma=W
$$

when written in terms of Wess-Zumino derivatives. Using conformal superspace derivatives, this constraint is simply

$$
-\frac{1}{4} \bar{\nabla}^{2} \Gamma=W
$$


Recall that this action arose by performing a duality transformation on the action

$$
S=-3 \int \mathrm{d}^{4} x \mathrm{~d}^{4} \theta E \phi \bar{\phi} \mathrm{e}^{-K / 3}+\int \mathrm{d}^{4} x \mathrm{~d}^{2} \theta \mathcal{E} \phi^{3} W+\int \mathrm{d}^{4} x \mathrm{~d}^{2} \bar{\theta} \overline{\mathcal{E}} \bar{\phi}^{3} \bar{W} .
$$

There exists a quite elegant formulation for dealing with this model, known as Kähler superspace, which was constructed originally in [6] 16 As a first step toward understanding how to geometrize the dual action, we will describe how Kähler superspace arises via the compensator method.

The first step in this procedure is to identify the function $K$ as a (composite) prepotential for the symmetry $U(1)_{K}$. The superfields $W$ and $\phi$ are naturally understood as sections transforming under this symmetry and are conventionally chiral with respect to it. We may introduce covariantly chiral superfields $\Phi$ and $\mathcal{W}$, which in the Hermitian basis are defined by

$$
\Phi:=\mathrm{e}^{-K / 6} \phi, \quad \mathcal{W}:=\mathrm{e}^{K / 2} W \quad \text { (Hermitian basis) }
$$

This requires that we introduce a $U(1)_{K}$ connection into our covariant derivative; for the conformal superspace derivative, we have

$$
\nabla_{A}^{\prime}=\nabla_{A}-\mathrm{i} A_{A}^{(K)} \mathbf{K}
$$

(henceforth dropping the prime) where the $U(1)_{K}$ generator $\mathbf{K}$ acts on $\Phi$ and $\mathcal{W}$ as

$$
\mathbf{K} \Phi=-\frac{2}{3} \Phi, \quad \mathbf{K} \mathcal{W}=2 \mathcal{W}
$$

In the Hermitian basis, the new connection is given by

$$
A_{\alpha}^{(K)}=\frac{\mathrm{i}}{4} \nabla_{\alpha} K, \quad \bar{A}_{\dot{\alpha}}^{(K)}=-\frac{\mathrm{i}}{4} \bar{\nabla}_{\dot{\alpha}} K, \quad A_{\alpha \dot{\alpha}}^{(K)}=\frac{1}{8}\left[\nabla_{\alpha}, \bar{\nabla}_{\dot{\alpha}}\right] K \quad \text { (Hermitian basis) }
$$

In any basis, the action becomes

$$
S=-3 \int \mathrm{d}^{4} x \mathrm{~d}^{4} \theta E \Phi \bar{\Phi}+\int \mathrm{d}^{4} x \mathrm{~d}^{2} \theta \mathcal{E} \Phi^{3} \mathcal{W}+\int \mathrm{d}^{4} x \mathrm{~d}^{2} \bar{\theta} \overline{\mathcal{E}} \bar{\Phi}^{3} \overline{\mathcal{W}}
$$

where the Kähler potential is now implicit in the chirality constraint on $\Phi$

$$
\bar{\nabla}_{\dot{\alpha}} \Phi=0
$$

The covariant derivatives of conformal superspace, augmented with a $U(1)_{K}$ connection, now obey the constraints (5.8a) with (5.8b) modified to read

$$
\left[\nabla_{\beta}, \nabla_{\alpha \dot{\alpha}}\right]=\frac{\mathrm{i}}{2} \varepsilon_{\beta \alpha} \bar{K}_{\dot{\alpha}} \mathbf{K}+\ldots, \quad \bar{K}_{\dot{\alpha}}:=-\frac{1}{4} \nabla^{2} \bar{\nabla}_{\dot{\alpha}} K
$$

\footnotetext{
${ }^{16}$ See [7] for a recent detailed and pedagogical discussion.
} 
where the ellipsis denotes the previous set of terms in (5.8b).

We now proceed to apply the compensator procedure as outlined in section 5 . However, there is one crucial difference. Before, the complex compensator $\mathbb{F}$ transformed only under dilatations and $U(1)_{R}$ transformations. Since here we wish to take $\mathbb{F}=\Phi^{1 / 2} \bar{\Phi}^{-1}$, it will transform under dilatations, $U(1)_{R}$, and $U(1)_{K}$ transformations. By examining the $U(1)_{K}$ and $U(1)_{R}$ charges of $\Phi$, we see that

$$
(\mathbf{K}+\mathbf{A}) \Phi=0 \Longrightarrow(\mathbf{K}+\mathbf{A}) \mathbb{F}=0
$$

Thus there is no reason why curvatures involving the combination $\mathbf{K}+\mathbf{A}$ cannot appear in $\left[\mathbb{D}_{A}, \mathbb{D}_{B}\right]$. In fact, we find that they do 17 An explicit calculation yields

$$
\left[\mathbb{D}_{\beta}, \mathbb{D}_{\alpha \dot{\alpha}}\right]=\frac{\mathrm{i}}{2} \varepsilon_{\beta \alpha} \overline{\mathbb{K}}_{\dot{\alpha}}(\mathbf{A}+\mathbf{K})+\ldots
$$

where the ellipsis refers to terms we had before. We still have

$$
\mathbb{T}_{\alpha}=\mathbb{F} \nabla_{\alpha} \log \left(\mathbb{F}^{4} \overline{\mathbb{F}}^{2}\right)=-3 \mathbb{F} \nabla_{\alpha} \log \bar{\Phi}=0
$$

as in Wess-Zumino superspace. It turns out that the algebra is (nearly) identical to the algebra of $U(1)$ superspace, but with the generator $\mathbf{A}$ replaced by $\mathbf{A}+\mathbf{K}$ and the curvature superfield $\mathbf{X}_{\alpha}$ identified with $\mathbb{K}_{\alpha}$, reflecting the fact that only the group $U(1)_{R+K}$ is gauged. In fact, the generator $\mathbf{A}$ may even be dropped from the combination $\mathbf{A}+\mathbf{K}$ since there are no longer any objects in the theory with $U(1)_{R}$ weight; they have all been modified with appropriate factors of the complex compensator to be $U(1)_{R}$ inert. Essentially, the complex compensator has transmuted

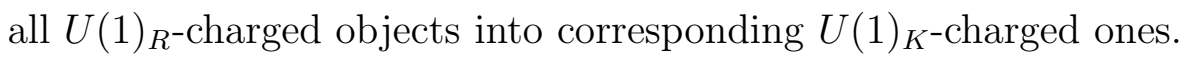

Armed with this insight, we may consider now the geometry associated with a complex linear multiplet carrying Kähler weight. This appears naturally if we consider the dual of the model (6.9). In first order form, we introduce the action

$$
S=\int \mathrm{d}^{4} x \mathrm{~d}^{4} \theta E\left(-3 \Phi \bar{\Phi}+\Gamma \Phi^{3}+\bar{\Gamma} \bar{\Phi}^{3}\right)
$$

where $\Phi$ and $\Gamma$ transform covariantly under both $U(1)_{K}$ and $U(1)_{R}$. $\Phi$ is otherwise an unconstrained complex superfield while $\Gamma$ obeys

$$
-\frac{1}{4} \bar{\nabla}^{2} \Gamma=\mathcal{W}
$$

\footnotetext{
${ }^{17}$ In particle physics parlance, $\Phi$ has "higgsed" the group $U(1)_{R} \times U(1)_{K}$ to the unbroken subgroup $U(1)_{R+K}$.
} 
where $\mathcal{W}$ is the Kähler-covariant superpotential. The equation of motion for $\Gamma$ enforces that $\Phi$ is covariantly chiral. Integrating out $\Phi$ instead using

$$
\Phi^{3}=\Gamma^{-2} \bar{\Gamma}^{-1}
$$

gives the dual action

$$
S_{\text {dual }}=-\int \mathrm{d}^{4} x \mathrm{~d}^{4} \theta E(\Gamma \bar{\Gamma})^{-1}
$$

with $\Gamma$ obeying the constraint (6.16).

If we now choose the complex compensator $\mathbb{F}=\bar{\Gamma}^{1 / 2}$ we find the algebra

$$
\begin{aligned}
\left\{\mathbb{D}_{\beta}, \mathbb{D}_{\alpha}\right\}= & -4 \overline{\mathbb{R}} M_{\beta \alpha}, \quad\left\{\overline{\mathbb{D}}^{\dot{\beta}}, \overline{\mathbb{D}}^{\dot{\alpha}}\right\}=4 \mathbb{R}^{\dot{\beta} \dot{\alpha}}, \quad\left\{\mathbb{D}_{\alpha}, \overline{\mathbb{D}}_{\dot{\alpha}}\right\}=-2 \mathrm{i} \mathbb{D}_{\alpha \dot{\alpha}} \\
{\left[\mathbb{D}_{\beta}, \mathbb{D}_{\alpha \dot{\alpha}}\right]=} & \frac{1}{2} \varepsilon_{\beta \alpha} \mathbb{T}^{\gamma} \mathbb{D}_{\gamma \dot{\alpha}}+\mathrm{i} \varepsilon_{\beta \alpha}\left(\overline{\mathbb{R}}+\frac{1}{8} \mathbb{D}^{\gamma} \mathbb{T}_{\gamma}-\frac{1}{16} \mathbb{T}^{\gamma} \mathbb{T}_{\gamma}\right) \overline{\mathbb{D}}_{\dot{\alpha}} \\
& -\mathrm{i} \varepsilon_{\beta \alpha}\left(\mathbb{G}_{\gamma \dot{\alpha}}+\frac{1}{8} \mathbb{D}_{\gamma} \overline{\mathbb{T}}_{\dot{\alpha}}+\frac{1}{8} \overline{\mathbb{D}}_{\dot{\alpha}} \mathbb{T}_{\gamma}\right) \mathbb{D}^{\gamma}+\mathrm{i} \overline{\mathbb{D}}_{\dot{\alpha}} \overline{\mathbb{R}}_{\beta \alpha} \\
& -\mathrm{i} \varepsilon_{\beta \alpha}\left(\mathbb{D}_{\delta}-\frac{1}{2} \mathbb{T}_{\delta}\right)\left(\mathbb{G}_{\gamma \dot{\alpha}}+\frac{1}{8} \mathbb{D}_{\gamma} \overline{\mathbb{T}}_{\dot{\alpha}}+\frac{1}{8} \overline{\mathbb{D}}_{\dot{\alpha}} \mathbb{T}_{\gamma}\right) M^{\gamma \delta} \\
& +2 \mathrm{i} \varepsilon_{\beta \alpha} \overline{\mathbb{W}}_{\dot{\alpha} \dot{\beta} \dot{\gamma}} \bar{M}^{\dot{\beta} \gamma}-2 \mathrm{i} \varepsilon_{\beta \alpha} \overline{\mathbb{Y}}^{\dot{\beta}} \bar{M}_{\dot{\beta} \dot{\alpha}}+\frac{\mathrm{i}}{2} \varepsilon_{\beta \alpha} \overline{\mathbb{K}}_{\dot{\alpha}}(\mathbf{A}+\mathbf{K})
\end{aligned}
$$

Because all objects carrying $U(1)_{R}$ charge have been modified with factors of the compensator so that they now carry only $U(1)_{K}$ charge, we may dispense with the generator $\mathbf{A}$ in the above algebra if we like. Making use of (5.45), we find the constraint

$$
\mathbb{R}=\mathcal{W}
$$

The Kähler potential and superpotential have been completely absorbed into the geometry of superspace. In addition we have the simple action

$$
S_{\text {dual }}=-\int \mathrm{d}^{4} x \mathrm{~d}^{4} \theta \mathbb{E}
$$

containing non-minimal supergravity, the matter kinetic terms, and the matter superpotential.

This formulation can be naturally understood as the dual of the original Kähler superspace. There one has the geometric constraint $\mathbb{T}_{\alpha}=0$ and (one can show [7]) the compensator equation of motion $\mathbb{R}=\mathcal{W}$. In the dual formulation we have just constructed, we have the reverse: $\mathbb{R}=\mathcal{W}$ is a constraint while (one can show) $\mathbb{T}_{\alpha}=0$ becomes the compensator equation of motion. This is exactly what is expected in a dual formulation. 


\section{Concluding remarks and AdS supercurrents}

The dual formulation we have constructed is quite interesting, if only because it resolves the long-standing puzzle of finding an AdS formulation of non-minimal supergravity which linearizes to the transverse superspin-3/2 model in AdS, eq. (A.5). We have also shown one way to see why only the $n=-1$ version of non-minimal supergravity has such a linearized action: it is the only version of supergravity for which we can deform the constraint $\left(\overline{\mathcal{D}}^{2}-4 R\right) \Sigma=0$ to $\left(\overline{\mathcal{D}}^{2}-4 R\right) \Gamma=-4 \mu=\mathrm{const}$ in a super-Weyl invariant way 18

More importantly, however, it emphasizes a key feature of AdS which we discussed in [1]: there are two irreducible supercurrents, and each one corresponds to some formulation of supergravity in AdS, either old minimal or non-minimal. The supercurrent associated with old minimal supergravity is the Ferrara-Zumino multiplet [34], obeying

$$
\overline{\mathcal{D}}^{\dot{\alpha}} J_{\alpha \dot{\alpha}}=\mathcal{D}_{\alpha} X, \quad \overline{\mathcal{D}}_{\dot{\alpha}} X=0
$$

where $J_{\alpha \dot{\alpha}}$ is real and $X$ is chiral. These superfields may be calculated in any model by varying the action with respect to the prepotential $H_{\alpha \dot{\alpha}}$ and the compensator $\phi$,

$$
J_{\alpha \dot{\alpha}}=\frac{\delta S}{\delta H^{\dot{\alpha} \alpha}}, \quad X=-\frac{1}{3} \frac{\delta S}{\delta \phi} .
$$

The supercurrent associated with non-minimal supergravity i. 19

$$
\overline{\mathcal{D}}^{\dot{\alpha}} J_{\alpha \dot{\alpha}}=-\frac{1}{4} \overline{\mathcal{D}}^{2} \zeta_{\alpha}, \quad \mathcal{D}_{(\beta} \zeta_{\alpha)}=0
$$

where $\zeta_{\alpha}$ is calculated by

$$
\zeta_{\alpha}=\frac{\delta S}{\delta \psi^{\alpha}}
$$

The superfield $\psi_{\alpha}$ denotes a prepotential for $\bar{\Gamma}$,

$$
\bar{\Gamma}=\mathcal{D}^{\alpha} \psi_{\alpha}+\cdots
$$

\footnotetext{
${ }^{18}$ For a general $n \neq-1$, we can consider a deformation $\left(\overline{\mathcal{D}}^{2}-4 R\right) \Gamma=-4 Q$ where $Q$ is a chiral scalar with nontrivial super-Weyl transformation.

${ }^{19}$ Actually, there is a broader class of non-minimal supercurrents associated with the ability to redefine $\Gamma$ in the linearized action (A.5) as follows $\Gamma \rightarrow \Gamma+\lambda \overline{\mathcal{D}}_{\dot{\alpha}} \mathcal{D}_{\alpha} H^{\alpha \dot{\alpha}}$, for a complex parameter $\lambda$. The choice implicitly made here corresponds to the simplest form of the linearized action.
} 
where the ellipsis denotes matter contributions 20 The constraint $\mathcal{D}_{(\beta} \zeta_{\alpha)}=0$ arises because $\psi_{\alpha}$ possesses the gauge invariance $\delta \psi_{\alpha}=\mathcal{D}^{\beta} \Omega_{\beta \alpha}$ for $\Omega_{\beta \alpha}=\Omega_{\alpha \beta}$. Equivalently, if the action is written only in terms of $\Gamma$ and $\bar{\Gamma}$, then

$$
\zeta_{\alpha}=\frac{\delta S}{\delta \psi^{\alpha}}=-\mathcal{D}_{\alpha} \frac{\delta S}{\delta \bar{\Gamma}}=\mathcal{D}_{\alpha}(V+\mathrm{i} U)
$$

for real well-defined operators $V$ and $U 21$

It is easy to check that the AdS supercurrents are related to each other by welldefined improvement transformations. The construction is a simple AdS generalization of that given in [35] for the Minkowski case. Beginning with the non-minimal supercurrent (7.3), one can construct

$$
\hat{J}_{\alpha \dot{\alpha}}=J_{\alpha \dot{\alpha}}+\frac{1}{6}\left[\mathcal{D}_{\alpha}, \overline{\mathcal{D}}_{\dot{\alpha}}\right] V-\mathcal{D}_{\alpha \dot{\alpha}} U, \quad \hat{X}:=\frac{1}{12}\left(\overline{\mathcal{D}}^{2}-4 \mu\right)(V-3 \mathrm{i} U) .
$$

It is a simple calculation to show that

$$
\overline{\mathcal{D}}^{\dot{\alpha}} \hat{J}_{\alpha \dot{\alpha}}=\mathcal{D}_{\alpha} \hat{X}
$$

(The reverse relation may also be easily constructed in a similar way.) It is easy to see that this improvement transformation matches the Minkowski case which arises by setting $\mu=0$. However, there is another purely AdS feature which we can exploit:

$$
\frac{1}{4 \mu} \overline{\mathcal{D}}^{2} \mathcal{D}_{\alpha} X=\mathcal{D}_{\alpha} X
$$

when $X$ is chiral, $\overline{\mathcal{D}}_{\dot{\alpha}} X=0$. This allows us to absorb any contribution $\mathcal{D}_{\alpha} X$ into the term $\zeta_{\alpha}$ via the redefinition

$$
\zeta_{\alpha}^{\prime}=\zeta_{\alpha}-\frac{1}{\mu} \mathcal{D}_{\alpha} X
$$

What is remarkable about this transformation is that it requires no assumptions about the global definition of the superfield $X$ itself; this transformation is globally defined so long as the original trace multiplet is globally defined.

In Minkowski space, it has recently been argued that the $\mathcal{S}$-multiplet [36]

$$
\bar{D}^{\dot{\alpha}} \mathcal{S}_{\alpha \dot{\alpha}}=\chi_{\alpha}+D_{\alpha} X, \quad \bar{D}_{\dot{\alpha}} \chi_{\alpha}=\bar{D}_{\dot{\alpha}} X=D^{\alpha} \chi_{\alpha}-\bar{D}_{\dot{\alpha}} \bar{\chi}^{\dot{\alpha}}=0
$$

\footnotetext{
${ }^{20}$ In other words, the first term $\mathcal{D}^{\alpha} \psi_{\alpha}$ corresponds to a complex linear superfield $\bar{\Sigma}$ obeying the homogeneous linearity condition $\left(\mathcal{D}^{2}-4 \bar{R}\right) \bar{\Sigma}=0$. It is this piece which we can vary independently of the matter fields.

${ }^{21}$ It is important to note that in AdS the equation $\mathcal{D}_{(\beta} \zeta_{\alpha)}=0$ can always be solved by $\zeta_{\alpha}=\mathcal{D}_{\alpha} \zeta$ for a complex globally-defined superfield which can be chosen as $\zeta=\mathcal{D}^{\gamma} \zeta_{\gamma} / 4 \bar{\mu}$. This is a major difference from Minkowski space where one cannot guarantee a globally-defined $\zeta$ always exists.
} 
and its natural generalization given in [37] is the most general (although it does not correspond to any known irreducible $\mathcal{N}=1$ supergravity formulation). Our interpretation of this statement is that that any other supercurrent which appears in a particular supersymmetric field theory in Minkowski space may be related to the $\mathcal{S}$-multiplet by a well-defined improvement transformation. It is worth pointing out that the $\mathcal{S}$-multiplet is extremely natural in $\mathcal{N}=2$ supersymmetric field theories [1] as it is contained within the $\mathcal{N}=2$ supercurrent which we constructed in [38]. However, the extension of the $\mathcal{S}$-multiplet to $\mathcal{N}=1$ AdS is unclear [1]. As we have discussed there are only two irreducible supercurrent multiplets in AdS.

As a simple illustration of AdS supercurrents, we consider the case of the most general non-linear sigma model in AdS,

$$
S=\int \mathrm{d}^{4} x \mathrm{~d}^{4} \theta E K+\int \mathrm{d}^{4} x \mathrm{~d}^{2} \theta \mathcal{E} W_{\mathrm{AdS}}+\int \mathrm{d}^{4} x \mathrm{~d}^{2} \bar{\theta} \overline{\mathcal{E}} \bar{W}_{\mathrm{AdS}}
$$

which can be rewritten using the AdS version of (3.12) as

$$
S=\int \mathrm{d}^{4} x \mathrm{~d}^{4} \theta E \mathcal{K}, \quad \mathcal{K}:=K+\frac{W_{\mathrm{AdS}}}{\mu}+\frac{\bar{W}_{\mathrm{AdS}}}{\bar{\mu}} .
$$

The model is invariant under Kähler transformations of the form

$$
K \rightarrow K+F+\bar{F}, \quad W_{\mathrm{AdS}} \rightarrow W_{\mathrm{AdS}}-\mu F
$$

for which the combination $\mathcal{K}$ is inert. This action may be understood as the $\kappa \rightarrow 0$ limit of the supergravity model

$$
S=-\frac{3}{\kappa^{2}} \int \mathrm{d}^{4} x \mathrm{~d}^{4} \theta E \phi \bar{\phi} \mathrm{e}^{-\kappa^{2} K / 3}+\int \mathrm{d}^{4} x \mathrm{~d}^{2} \theta \mathcal{E} \phi^{3} W+\int \mathrm{d}^{4} x \mathrm{~d}^{2} \bar{\theta} \overline{\mathcal{E}} \bar{\phi}^{3} \bar{W}
$$

if we encode the AdS superpotential into the supergravity superpotential $W$ via

$$
W=\frac{\mu}{\kappa^{2}} \exp \left(\kappa^{2} W_{\mathrm{AdS}} / \mu\right)
$$

This identification is useful since then the Kähler transformations have exactly the same form in both the supergravity formulation and in the AdS limit.

In the full supergravity background in the gauge where $\phi=1$, the supercurrent equation can be written

$$
\overline{\mathcal{D}}^{\dot{\alpha}} J_{\alpha \dot{\alpha}}=\mathcal{D}_{\alpha} X
$$


where the derivatives $\mathcal{D}_{A}$ are the usual Wess-Zumino superspace derivatives. The real superfield $J_{\alpha \dot{\alpha}}$ and the chiral superfield $X$ are given respectively by

$$
\begin{aligned}
J_{\alpha \dot{\alpha}} & :=\frac{1}{\kappa^{2}} \mathrm{e}^{-\kappa^{2} K / 3}\left(G_{\alpha \dot{\alpha}}+\mathrm{e}^{-\kappa^{2} K / 6}\left[\mathcal{D}_{\alpha}, \overline{\mathcal{D}}_{\dot{\alpha}}\right] \mathrm{e}^{\kappa^{2} K / 6}-\frac{\kappa^{2}}{2} K_{i \bar{j}} \mathcal{D}_{\alpha} \phi^{i} \overline{\mathcal{D}}_{\dot{\alpha}} \bar{\phi}^{\bar{j}}\right) \\
& =\mathrm{e}^{-\kappa^{2} K / 3}\left(\frac{1}{\kappa^{2}} G_{\alpha \dot{\alpha}}+\frac{1}{6}\left[\mathcal{D}_{\alpha}, \overline{\mathcal{D}}_{\dot{\alpha}}\right] K+\frac{\kappa^{2}}{18} \mathcal{D}_{\alpha} K \overline{\mathcal{D}}_{\dot{\alpha}} K-\frac{1}{2} K_{i \bar{j}} \mathcal{D}_{\alpha} \phi^{i} \overline{\mathcal{D}}_{\dot{\alpha}} \bar{\phi}^{\bar{j}}\right) \\
X & :=-\frac{1}{4 \kappa^{2}}\left(\overline{\mathcal{D}}^{2}-4 R\right) \mathrm{e}^{-\kappa^{2} K / 3}-W \\
& =\mathrm{e}^{-\kappa^{2} K / 3}\left(\frac{1}{\kappa^{2}} R+\frac{1}{12} \overline{\mathcal{D}}^{2} K-\frac{\kappa^{2}}{36} \overline{\mathcal{D}}_{\dot{\alpha}} K \overline{\mathcal{D}}^{\dot{\alpha}} K\right)-W
\end{aligned}
$$

This form involves operators which are not Kähler invariant, but this is no surprise; in the frame $\phi=1$, every Kähler transformation must be accompanied by a super-Weyl transformation to restore invariance. One is tempted to identify the first term of $J_{\alpha \dot{\alpha}}$ and of $X$ as the "supergravity term" and the rest as "matter contributions;" however, this is completely dependent on the way one defines the supergeometry. For example, if we exchange Wess-Zumino superspace geometry for Kähler superspace geometry, the supercurrent equation takes the form

$$
\overline{\mathbb{D}}^{\dot{\alpha}} J_{\alpha \dot{\alpha}}=\mathbb{D}_{\alpha} X
$$

where

$$
J_{\alpha \dot{\alpha}}:=\frac{1}{\kappa^{2}} \mathbb{G}_{\alpha \dot{\alpha}}-\frac{1}{2} K_{i \bar{j}} \mathbb{D}_{\alpha} \phi^{i} \overline{\mathbb{D}}_{\dot{\alpha}} \bar{\phi}^{\bar{j}}, \quad X:=\frac{1}{\kappa^{2}} \mathbb{R}-\mathcal{W}
$$

Here $\mathbb{D}_{A}$ is the covariant derivative and $\mathbb{G}_{\alpha \dot{\alpha}}$ and $\mathbb{R}$ the torsion tensors in the Kähler superspace formulation. This naively has the Ferrara-Zumino form and the first terms of both $J_{\alpha \dot{\alpha}}$ and $X$ involve what appear to be purely supergravity terms. However, this is misleading as $\mathbb{R}$ and $\mathbb{G}$ obey the Kähler superspace constraint

$$
\mathbb{D}_{\alpha} \mathbb{R}-\overline{\mathbb{D}}^{\dot{\alpha}} \mathbb{G}_{\alpha \dot{\alpha}}=-\frac{\kappa^{2}}{4}\left(\overline{\mathbb{D}}^{2}-4 \mathbb{R}\right) \mathbb{D}_{\alpha} K
$$

which is decidedly a matter coupling. Making use of this identity, the supercurrent equation can be recast in the form

$$
-\frac{1}{2} \overline{\mathbb{D}}^{\dot{\alpha}} \mathbb{K}_{\alpha \dot{\alpha}}=\mathbb{K}_{\alpha}-\mathbb{D}_{\alpha} \mathcal{W}
$$

where

$$
\mathbb{K}_{\alpha \dot{\alpha}}:=K_{i \bar{j}} \mathbb{D}_{\alpha} \phi^{i} \overline{\mathbb{D}}_{\dot{\alpha}} \bar{\phi}^{\bar{j}}, \quad \mathbb{K}_{\alpha}:=-\frac{1}{4}\left(\overline{\mathbb{D}}^{2}-4 \mathbb{R}\right) \mathbb{D}_{\alpha} K
$$


This equation holds on-shell due to the matter equation of motion

$$
\frac{1}{4} \overline{\mathbb{D}}^{2} K_{i}=\nabla_{i} \mathcal{W}
$$

where in the Hermitian basis

$$
\left.\nabla_{i} \mathcal{W}:=\mathrm{e}^{\kappa^{2}\left(K / 2+W_{\mathrm{AdS}} / \mu\right)} \partial_{i}\left(W_{\mathrm{AdS}}+\mu K\right) \quad \text { (Hermitian basis }\right)
$$

The supercurrent (7.22) has the form of the $\mathcal{S}$-multiplet but again this is somewhat misleading since $\mathcal{W}$ is covariant with respect to Kähler transformations and the derivatives carry a Kähler connection. When we take the $\kappa \rightarrow 0$ limit, we recover (being careful to include the contribution from the $U(1)_{K}$ connection)

$$
-\frac{1}{2} \overline{\mathcal{D}}^{\dot{\alpha}} K_{\alpha \dot{\alpha}}=K_{\alpha}-\mathcal{D}_{\alpha}\left(W_{\mathrm{AdS}}+\mu K\right)
$$

where

$$
K_{\alpha \dot{\alpha}}:=K_{i \bar{j}} \mathcal{D}_{\alpha} \phi^{i} \overline{\mathcal{D}}_{\dot{\alpha}} \bar{\phi}^{\bar{j}}, \quad K_{\alpha}:=-\frac{1}{4}\left(\overline{\mathcal{D}}^{2}-4 \mu\right) \mathcal{D}_{\alpha} K
$$

with the derivatives $\mathcal{D}_{A}$ obeying the AdS superspace algebra (4.14). This is still not quite the $\mathcal{S}$-multiplet due to the non-chiral term $\mu K$ appearing in the supercurrent equation 22 On the other hand, we observe that the equation may be rewritten in the form

$$
\begin{aligned}
-\frac{1}{2} \overline{\mathcal{D}}^{\dot{\alpha}} K_{\alpha \dot{\alpha}} & =-\frac{1}{4} \overline{\mathcal{D}}^{2} \mathcal{D}_{\alpha} K-\mathcal{D}_{\alpha} W_{\mathrm{AdS}} \\
& =-\frac{1}{4} \overline{\mathcal{D}}^{2} \mathcal{D}_{\alpha}\left(K+\frac{W_{\mathrm{AdS}}}{\mu}+\frac{\bar{W}_{\mathrm{AdS}}}{\bar{\mu}}\right)=-\frac{1}{4} \overline{\mathcal{D}}^{2} \mathcal{D}_{\alpha} \mathcal{K}
\end{aligned}
$$

where $\mathcal{K}$ is the globally defined Lagrangian for the general non-linear sigma model in AdS. This has precisely the form of the non-minimal $(n=-1)$ supercurrent conservation equation and one may check by an explicit calculation that it holds due to the matter equation of motion in AdS,

$$
\frac{1}{4} \overline{\mathcal{D}}^{2} K_{i}=\partial_{i}\left(W_{\mathrm{AdS}}+\mu K\right)=\mu \partial_{i} \mathcal{K} \Longrightarrow \frac{1}{4}\left(\overline{\mathcal{D}}^{2}-4 \mu\right) \mathcal{K}_{i}=0
$$

In light of these considerations we propose the following supercurrent formula as the general supercurrent in AdS

$$
\overline{\mathcal{D}}^{\dot{\alpha}} J_{\alpha \dot{\alpha}}=\mathcal{D}_{\alpha} X-\frac{1}{4} \overline{\mathcal{D}}^{2} \zeta_{\alpha}, \quad \overline{\mathcal{D}}_{\dot{\alpha}} X=\mathcal{D}_{(\beta} \zeta_{\alpha)}=0
$$

\footnotetext{
${ }^{22}$ This expression may still be interpreted as the $\mathcal{S}$-multiplet if one thinks of the $\mu K$ term as arising from the connection for the nonlinear Kähler transformation of $W_{\mathrm{AdS}}$, but this seems to violate the spirit of the $\mathcal{S}$-multiplet.
} 
Each of the trace multiplets, $X$ and $\zeta_{\alpha}$, may be identified with a specific linearized action in AdS, which due to the results of this paper, can both be extended to a full nonlinear action. While the inclusion of both trace multiplet contributions seems to imply this is a $24+24$ component supercurrent, we must keep in mind the symmetry

$$
X \rightarrow X+\mu \Lambda, \quad \zeta_{\alpha} \rightarrow \zeta_{\alpha}+\mathcal{D}_{\alpha} \Lambda, \quad \overline{\mathcal{D}}_{\dot{\alpha}} \Lambda=0
$$

which we exploited above. This reduces the supercurrent to $20+20$ components.

It is interesting that the AdS supercurrent (7.30) reduces in the naive Minkowski limit to a $20+20$ supercurrent of the form 23 ,

$$
\begin{gathered}
\bar{D}^{\dot{\alpha}} J_{\alpha \dot{\alpha}}=D_{\alpha} X+\chi_{\alpha}+\mathrm{i} \eta_{\alpha} \\
\bar{D}_{\dot{\alpha}} X=\bar{D}_{\alpha} \chi_{\alpha}=\bar{D}_{\dot{\alpha}} \eta_{\alpha}=D^{\alpha} \chi_{\alpha}-\bar{D}_{\dot{\alpha}} \bar{\chi}^{\dot{\alpha}}=D^{\alpha} \eta_{\alpha}-\bar{D}_{\dot{\alpha}} \bar{\eta}^{\dot{\alpha}}=0
\end{gathered}
$$

which arises from the consideration of all the possible models of linearized supergravity categorized in recent years (see [40, 35] and the references therein). It was argued in [37] that this more general supercurrent can always be improved to the $\mathcal{S}$-multiplet. The closest analogy for the proposed AdS supercurrent (7.30) would be the statement that $\zeta_{\alpha}$ may always be improved to $\zeta_{\alpha}=\mathcal{D}_{\alpha} U$ for real superfield $U$. If so, then the supercurrent (7.30) would be reduced to $16+16$ components in AdS and naturally approach the $\mathcal{S}$-multiplet form in the naive Minkowski limit.

\section{Acknowledgements:}

This work is supported in part by the Australian Research Council and by a UWA Research Development Award.

\section{A The massless superspin-3/2 multiplet in AdS}

Off-shell massless higher-superspin multiplets were constructed in AdS superspace in [2]. For the case of half-integer superspins, they are characterized by a family of dually equivalent models beginning at superspin-3/2. The so-called longitudinal formulation of the free massless superspin-3/2 multiplet in AdS can be written

$$
\begin{aligned}
S_{(3 / 2)}^{\|}= & -\int \mathrm{d}^{4} x \mathrm{~d}^{4} \theta E\left\{\frac{1}{16} H^{\dot{\alpha} \alpha} \mathcal{D}^{\beta}\left(\overline{\mathcal{D}}^{2}-4 R\right) \mathcal{D}_{\beta} H_{\alpha \dot{\alpha}}-\frac{1}{48}\left(\left[\mathcal{D}_{\alpha}, \overline{\mathcal{D}}_{\dot{\alpha}}\right] H^{\dot{\alpha} \alpha}\right)^{2}\right. \\
& \left.+\frac{1}{4}\left(\mathcal{D}_{\alpha \dot{\alpha}} H^{\dot{\alpha} \alpha}\right)^{2}+\frac{1}{4} R \bar{R} H^{\dot{\alpha} \alpha} H_{\alpha \dot{\alpha}}+\mathrm{i} H^{\dot{\alpha} \alpha} \mathcal{D}_{\alpha \dot{\alpha}}(\phi-\bar{\phi})+3\left(\phi \bar{\phi}-\phi^{2}-\bar{\phi}^{2}\right)\right\} .
\end{aligned}
$$

\footnotetext{
${ }^{23}$ This supercurrent was considered in a slightly different form in [39].
} 
The superfield $H_{\alpha \dot{\alpha}}$ is real while $\phi$ is covariantly chiral,

$$
\overline{\mathcal{D}}_{\dot{\alpha}} \phi=0
$$

The action possesses the gauge invariance

$$
\delta H_{\alpha \dot{\alpha}}=\mathcal{D}_{\alpha} \bar{L}_{\dot{\alpha}}-\overline{\mathcal{D}}_{\dot{\alpha}} L_{\alpha}, \quad \delta \phi=-\frac{1}{12}\left(\overline{\mathcal{D}}^{2}-4 R\right) \mathcal{D}^{\alpha} L_{\alpha}
$$

This is exactly the linearized action of old minimal supergravity with a cosmological term.

This model may be dualized to one involving a complex linear multiplet $\Gamma$, obeying

$$
\left(\overline{\mathcal{D}}^{2}-4 R\right) \Gamma=0
$$

The dual action, which is called the transverse formulation in the context of [2], is

$$
\begin{aligned}
S_{(3 / 2)}^{\perp}= & -\int \mathrm{d}^{4} x \mathrm{~d}^{4} \theta E\left\{\frac{1}{16} H^{\dot{\alpha} \alpha} \mathcal{D}^{\beta}\left(\overline{\mathcal{D}}^{2}-4 R\right) \mathcal{D}_{\beta} H_{\alpha \dot{\alpha}}+\frac{1}{4} R \bar{R} H^{\dot{\alpha} \alpha} H_{\alpha \dot{\alpha}}\right. \\
& \left.+\frac{1}{2} H^{\alpha \dot{\alpha}}\left(\mathcal{D}_{\alpha} \overline{\mathcal{D}}_{\dot{\alpha}} \Gamma-\overline{\mathcal{D}}_{\dot{\alpha}} \mathcal{D}_{\alpha} \bar{\Gamma}\right)+\bar{\Gamma} \Gamma+\Gamma^{2}+\bar{\Gamma}^{2}\right\},
\end{aligned}
$$

with the gauge invariance

$$
\delta H_{\alpha \dot{\alpha}}=\mathcal{D}_{\alpha} \bar{L}_{\dot{\alpha}}-\overline{\mathcal{D}}_{\dot{\alpha}} L_{\alpha}, \quad \delta \Gamma=-\frac{1}{4} \overline{\mathcal{D}}_{\dot{\alpha}} \mathcal{D}^{2} \bar{L}^{\dot{\alpha}}
$$

\section{References}

[1] D. Butter and S. M. Kuzenko, "N=2 AdS supergravity and supercurrents," arXiv:1104.2153 [hep-th].

[2] S. M. Kuzenko and A. G. Sibiryakov, "Free massless higher superspin superfields on the anti-de Sitter superspace," Phys. Atom. Nucl. 57, 1257 (1994) [Yad. Fiz. 57, 1326 (1994)].

[3] S. J. Gates Jr., M. T. Grisaru, M. Roček and W. Siegel, Superspace, or One Thousand and One Lessons in Supersymmetry, Benjamin/Cummings (Reading, MA), 1983, hep-th/0108200

[4] I. L. Buchbinder and S. M. Kuzenko, Ideas and Methods of Supersymmetry and Supergravity or a Walk Through Superspace, IOP, Bristol, 1998.

[5] B. B. Deo and S. J. Gates Jr., "Non-minimal N=1 supergravity and broken global supersymmetry," Nucl. Phys. B 151, 195 (1985).

[6] P. Binetruy, G. Girardi, R. Grimm and M. Müller, "Kähler transformations and the coupling of matter and Yang-Mills fields to supergravity," Phys. Lett. B 189, 83 (1987).

[7] P. Binetruy, G. Girardi and R. Grimm, "Supergravity couplings: a geometric formulation," Phys. Rept. 343, 255 (2001) arXiv:hep-th/0005225. 
[8] J. Wess and J. Bagger, Supersymmetry and Supergravity, Princeton University Press, Princeton, 1992.

[9] B. Zumino, "Superspace," in Unification of the Fundamental Particle Interactions, S. Ferrara, J. Ellis and P. van Nieuwenhuizen (Eds.), Plenum Press, 1980, p. 101.

[10] S. J. Gates Jr. and W. Siegel, "Variant superfield representations," Nucl. Phys. B 187, 389 (1981).

[11] W. Siegel and S. J. Gates Jr. "Superfield supergravity," Nucl. Phys. B 147, 77 (1979).

[12] S. M. Kuzenko and S. J. Tyler, "Complex linear superfield as a model for Goldstino," JHEP 1104, 057 (2011) [arXiv:1102.3042 [hep-th]].

[13] T. Kugo and S. Uehara, "Improved superconformal gauge conditions in the $\mathrm{N}=1$ supergravity Yang-Mills matter system," Nucl. Phys. B 222, 125 (1983).

[14] J. Wess and B. Zumino, "Superfield Lagrangian for supergravity," Phys. Lett. B 74, 51 (1978).

[15] K. S. Stelle and P. C. West, "Minimal auxiliary fields for supergravity," Phys. Lett. B 74, 330 (1978); S. Ferrara and P. van Nieuwenhuizen, "The auxiliary fields of supergravity," Phys. Lett. B 74, 333 (1978).

[16] P. S. Howe and R. W. Tucker, "Scale invariance in superspace," Phys. Lett. B 80, 138 (1978).

[17] B. B. Deo and S. J. Gates Jr., "Comments on nonminimal N=1 scalar multiplets," Nucl. Phys. B 254, 187 (1985).

[18] V. P. Akulov, D. V. Volkov and V. A. Soroka, "Generally covariant theories of gauge fields on superspace," Theor. Math. Phys. 31, 285 (1977); M. F. Sohnius and P. C. West, "An alternative minimal off-shell version of N=1 supergravity," Phys. Lett. B 105, 353 (1981).

[19] P. Breitenlohner, "A geometric interpretation of local supersymmetry," Phys. Lett. B 67, 49 (1977): "Some invariant Lagrangians for local supersymmetry," Nucl. Phys. B124, 500 (1977).

[20] S. Ferrara, L. Girardello, T. Kugo and A. Van Proeyen, "Relation between different auxiliary field formulations of N=1 supergravity coupled to matter," Nucl. Phys. B 223, 191 (1983).

[21] T. Kugo and S. Uehara, "N=1 superconformal tensor calculus: Multiplets with external indices and derivative operators," Prog. Theor. Phys. 73, 235 (1985).

[22] I. L. Buchbinder, S. M. Kuzenko and O. A. Soloviev, "One-loop counterterms of Wess-Zumino models in the N=1 non-minimal supergravity background," Nucl. Phys. B 322, 277 (1989).

[23] V. Ogievetsky and E. Sokatchev, "On vector superfield generated by supercurrent," Nucl. Phys. B 124, 309 (1977); "Structure of supergravity group," Phys. Lett. 79B, 222 (1978)

[24] W. Siegel, "Solution to constraints in Wess-Zumino supergravity formalism," Nucl. Phys. B 142, 301 (1978).

[25] M. Kaku, P. K. Townsend, "Poincare supergravity as broken superconformal gravity," Phys. Lett. B76, 54 (1978); M. Kaku, P. K. Townsend, P. van Nieuwenhuizen, "Properties of conformal supergravity," Phys. Rev. D17, 3179 (1978); P. K. Townsend, P. van Nieuwenhuizen, "Simplifications of conformal supergravity," Phys. Rev. D19, 3166 (1979); S. Ferrara, M. T. Grisaru, P. van Nieuwenhuizen, "Poincare and conformal supergravity models with closed algebras," 
Nucl. Phys. B138, 430 (1978); B. de Wit, M. Rocek, "Improved tensor multiplets," Phys. Lett. B109, 439 (1982); T. Kugo, S. Uehara, "Conformal and Poincare tensor calculi in N=1 supergravity," Nucl. Phys. B226, 49 (1983).

[26] P. Van Nieuwenhuizen, "Supergravity," Phys. Rept. 68, 189-398 (1981).

[27] E. S. Fradkin, A. A. Tseytlin, "Conformal supergravity," Phys. Rept. 119, 233-362 (1985).

[28] W. Siegel, "Superconformal invariance of superspace with nonminimal auxiliary fields," Phys. Lett. B 80, 224 (1979).

[29] S. J. Gates Jr. and W. Siegel, "Understanding constraints in superspace formulations of supergravity," Nucl. Phys. B 163, 519 (1980).

[30] S. J. Gates Jr., K. S. Stelle and P. C. West, "Algebraic origins of superspace constraints in supergravity," Nucl. Phys. B 169, 347 (1980).

[31] P. S. Howe, "Supergravity in superspace," Nucl. Phys. B 199, 309 (1982).

[32] D. Butter, "N=1 Conformal Superspace in Four Dimensions," Annals Phys. 325, 1026 (2010) arXiv:0906.4399 [hep-th]].

[33] D. Butter, "Background field formalism for chiral matter and gauge fields conformally coupled to supergravity," Nucl. Phys. B 828, 233 (2010) [arXiv:0909.4901 [hep-th]].

[34] S. Ferrara and B. Zumino, "Transformation properties of the supercurrent," Nucl. Phys. B 87, 207 (1975).

[35] S. M. Kuzenko, "Variant supercurrents and Noether procedure," Eur. Phys. J. C 71, 1513 (2011) arXiv:1008.1877 [hep-th]].

[36] Z. Komargodski and N. Seiberg, "Comments on supercurrent multiplets, supersymmetric field theories and supergravity," JHEP 1007, 017 (2010) [arXiv:1002.2228 [hep-th]].

[37] T. T. Dumitrescu and N. Seiberg, "Supercurrents and brane currents in diverse dimensions," arXiv:1106.0031 [hep-th].

[38] D. Butter and S. M. Kuzenko, "N=2 supergravity and supercurrents," JHEP 1012, 080 (2010) arXiv:1011.0339 [hep-th]].

[39] M. Magro, I. Sachs, S. Wolf, "Superfield Noether procedure," Annals Phys. 298, 123-166 (2002). hep-th/0110131.

[40] S. M. Kuzenko, "Variant supercurrent multiplets," JHEP 1004, 022 (2010) arXiv:1002.4932 [hep-th]]. 